\title{
Inventory constrained design of a timber funicular structure
}

\author{
Kim R. Baber ${ }^{1}\left[\right.$ ] Jane R. Burry ${ }^{2} \cdot$ Canhui Chen $^{2} \cdot$ Joseph M. Gattas ${ }^{3} \cdot$ Aurimas Bukauskas $^{4}$
}

Received: 2 December 2019 / Accepted: 7 August 2020 / Published online: 20 August 2020

(c) Springer Nature Switzerland AG 2020

\begin{abstract}
This research investigates the development of a digital form finding model that combines the generation of funicular geometry with a material inventory constraint. The model provides a flexible design tool that facilitates exploration of structural form whilst simultaneously satisfying two rationalizing criteria. It maintains an equilibrated structure derived from funicular geometry; and optimises the assignment of a unique inventory of timber members having natural dimensional variation. The combined goal for the design outcome is to achieve material efficiency through both structurally rational form and minimization of material waste. The material chosen for the inventory is utility-grade sawn timber, being lightweight but with high levels of naturally occurring structural variability. Sawn timber boards that are rejected for structural applications due to frequent structural defects (knots, checks, splits etc.) represent up to $50 \%$ of the sawn product produced by Australian sawmills, and are destined for under-valued non-structural use, chipping or burning. Yet these boards can readily yield usable short length structural members, once defects are removed. In doing so, the process creates a unique inventory of random short members. These short members are well suited to articulated structures, which, by employing an inverted funicular geometry, only incur axial stresses and can employ simple (non-moment resisting) timber connections. This form finding tool and a first prototype pavilion are proofs of concept for viable structural application of what is otherwise a significant source of waste in the timber industry.
\end{abstract}

Keywords Inventory-constrained design · Form finding · Funicular modelling · Timber structures

\section{Introduction}

\subsection{Utility-grade timber and inventory-constrained design}

There is considerable natural variation in the properties of trees harvested as feedstock for sawmills. This variation exists between plantation stands, between trees within the same stand, and within the tree itself, so the resultant distribution of defects within sawn timber is highly unpredictable and can only be evaluated after sawn board production. As a result, a large volume of boards are rendered uncertifiable for structural use, due to poor structural properties and/or a high incidence of natural defects. Ultimately, only about $50 \%$ of the volume of timber harvested will end up being certified for use as structural grade timber [1], shown in Fig. 1a-d, with remaining material sold as non-structural members, woodchipped to make particle based products, or burnt as fuel to power other operations in the sawmill plant.

For those timber members containing frequent defects such as knots, checks, splits, and wane [2], defects can be cut out and the resultant lengths of timber may be certified as structural grade, but are relatively short and

This research has been undertaken for the design, fabrication and installation of the pavilion for the "Working Group 21 - Advanced Manufacturing and Materials" exhibition at the IASS Symposium 2019.

Kim R. Baber, k.baber@uq.edu.au | 1 School of Architecture, University of Queensland, Brisbane, QLD 4067, Australia. ${ }^{2}$ School of Design, Swinburne University of Technology, Melbourne, VIC 3122, Australia. ${ }^{3}$ School of Civil Engineering, University of Queensland, Brisbane, QLD 4067, Australia. ${ }^{4}$ BRE Centre for Innovative Construction Materials, University of Bath, Bath BA2 7AY, UK. 


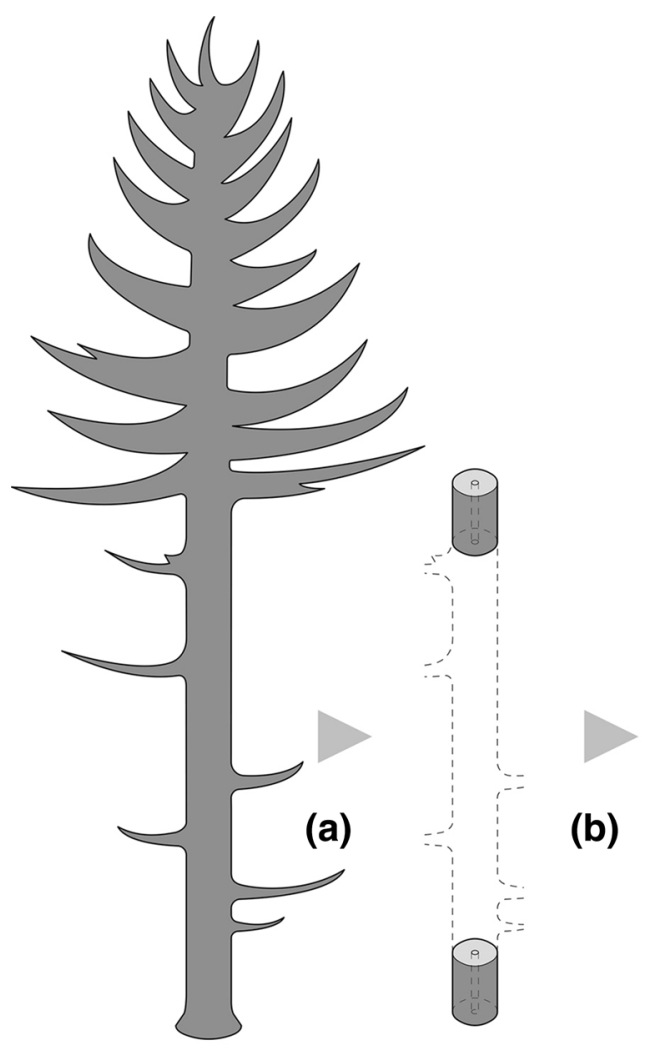

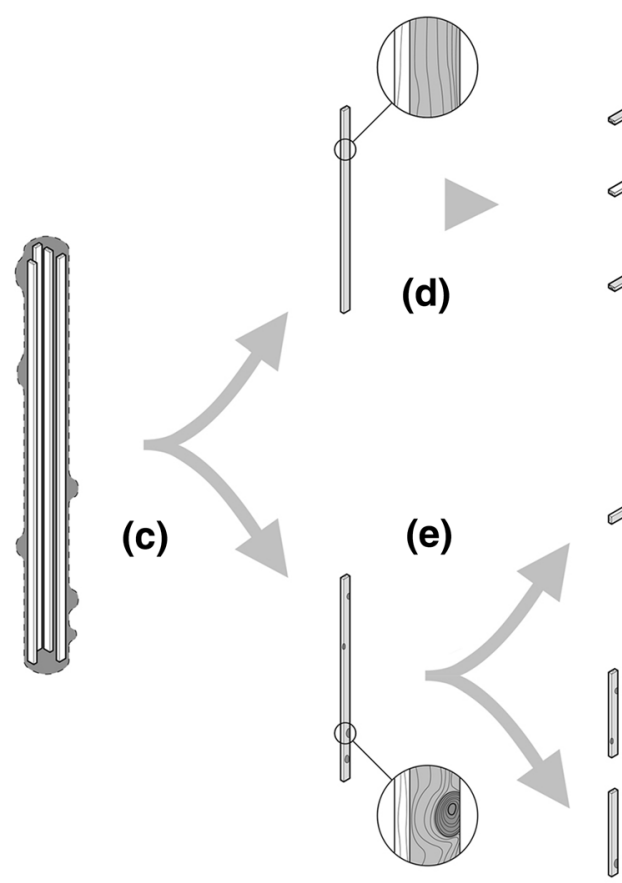

(f)
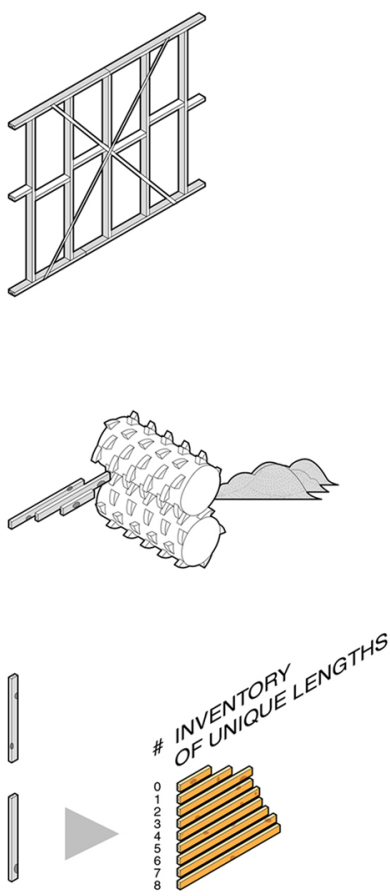

(g)
Fig. 1 A sawmilling process showing the generation of an inventory of unique lengths: a the tree is harvested and docked into transportable lengths (approx. $6 \mathrm{~m}$ ) with varying branch distribution according to: silvicultural practice, climate, soil, planting density etc.; $\mathbf{b}$ each log is sawn into usable framing members and is subject to the naturally occurring structural defects; c sawn mem-

inconsistent in length, as shown in Fig. 1e-g. For most Australian commercial framing applications, the variability contained in such a random inventory of elements is deemed too inconvenient for contractors to work with and consequently utility-grade products are often sold at a loss, below the price of the log itself. However, the increasing prominence of digital design and fabrication tools is eroding this limitation, with sophisticated workflows continuously emerging for handling of non-standard material inventories and design and assembly of mass-customised building products. Examples of complex freeform structures computationally assembled from short-length timber elements include the Bezier vault structure, with shape control achieved via element chamfer angles [3], and the Sequential Roof, with shape control achieved with variation of truss element lengths [4]. bers are sorted into those with and without defects; $\mathbf{d}$ members free from defects are certified for use in structural applications; e members with defects are used in non-structural application or woodchipped; $\mathbf{f}$ defects are removed, resulting in short lengths, which provide: $\mathbf{g}$ an inventory of short, unique lengths

While sophisticated processes exist for assembly of short length timber systems, computational approaches for problems where a designer must employ a constrained inventory of parts have only recently emerged. Such constraints arise for example with use of deconstructed building components [5] or use of natural material with high variation in sectional size or length [6]. Early methods assigned parts based on the geometric correspondence between an inventory of parts and a target form $[7,8]$. Among the first built structures resulting from such an approach was the Tree Fork Truss, for which forked trees were assigned to a Vierendeel truss geometry using an evolutionary algorithm [9]. State-of-the-art approaches use optimisation formulations that can assign parts based on a wide variety of possible fitness criteria, including structural form or topology, structural performance, 
minimisation of material waste, or minimisation of reprocessing expense $[5,6,10]$.

\subsection{Interactive funicular modelling}

Antoni Gaudís funicular or 'hanging chain' model was a sophisticated form finding tool for rationalizing structural geometry and optimising material utilization. Gaudís use of the tool in the design of the Colònia Güell church was particularly innovative, in that it involved a complex three-dimensional network of interconnected structural force vectors. Previous versions of hanging chain models, such as those used by Heinrich Hübsch were limited to discrete, two-dimensional studies of an arch structure in a single plane [11]. Gaudís hanging model proved to be highly effective in resolving complex geometric configurations of interconnecting structural elements into a state of equilibrium, in 'real time'.

As Block points out, physical hanging chain models, as used by Gaudí, are "excellent design explorers since they address both structural and formal considerations and present the designer at all times with a status of the design that reflects the cumulative changes applied to the model" [12]. They can be, however, incredibly time consuming and complex to build, and he states "the effect of removing, adding or adjusting the lengths of strings is not straightforward at all" [12]. This was experienced firsthand by Jos Tomlow and his team when they reconstructed Gaudís hanging chain model in 1989 [11].

Interactive computational hanging chain models, such as the CADenary project pioneered as a design tool by Axel Kilian, utilized particle spring systems to simulate virtual hanging chain models, allowing interaction with the model geometry in real time, providing immediate adjustment of the spring lengths within the chain as equilibrium is resolved [13]. The virtual model reacts in real time to changes. "[The] novelty of [Kilian's] implementation is to combine the dynamic simulation and interactive environment to find equilibrium and to allow the user to playfully explore these constantly evolving structures" [12]. For form finding models that work with branched and nested element structures, such as those developed by Hunt [14] and Hensel [15], of particular importance is the degree of interaction the model provides to the designer with regard to "specific local settings" [15], referring to the local parameters that finely-tuned the hierarchy, configuration and sizing of branches within the chain model.

This investigation seeks to include a specific local parameter in the funicular model through an additional criterion in the computational form-finding procedure. The model constrains the target chain lengths based on a local material inventory from which the final structure is to be constructed. In this case, it is based on naturally occurring short lengths of clear timber material available from boards with frequent defects. The aim is to introduce this additional criterion to a virtual form finding model, but ensure that the model operates with a similar level of interactive responsiveness, providing immediate spatial feedback: a design tool that facilitates exploration of form within the bounds of both structural and material constraints.

\subsection{IASS 'Working Group 21' competition}

The research undertaken in this project was applied in the design and fabrication of a full-scale prototype pavilion, entered in the 'Working Group 21: Advanced Manufacturing and Materials' competition organized by the International Association for Shell and Spatial Structures (IASS). This competition called for pavilion designs demonstrating innovation in the "construction of lightweight structures by cutting-edge techniques and smart materials for the built environment" to be exhibited and subsequently judged at the IASS Symposium in Barcelona, October 2019.

A limit on overall pavilion size was imposed as a cubic boundary of $4 \times 4 \times 4$ meters and a $192 \mathrm{~kg}$ maximum overall weight. Additional limitations on member sizes for transportability were also imposed: no element was to be larger than that capable of fitting in a box measuring $65 \mathrm{~cm} \times 75 \mathrm{~cm} \times 100 \mathrm{~cm}$, of which a maximum of 6 boxes were permitted. This size limit was an additional design constraint that was applied in conjunction with the constraints of the short utility grade timber members.

This paper presents a novel digital form-finding and inventory constrained design process that translates short utility grade timber members to a funicular spatial structure. Section 2 first presents the utility-grade length and funicular form generation and Sect. 3 then presents a fast heuristic for the assignment of a timber inventory to funicular locations, with form re-relaxation to suit the assigned part lengths. Sections 4 and 5 describe the fabrication, detailing, and construction of the full-scale pavilion built to validate the inventory-constrained funicular modelling process and meet the specific constraints imposed for the pavilion exhibition and competition.

\section{Short length timber as an inventory constraint}

\subsection{Material inventory representation}

A typical sawn board obtained from milling of an Australian softwood species may contain defects which cause it to fail a structural grading classification. Common defects include wane (missing portion of timber due to curvature 


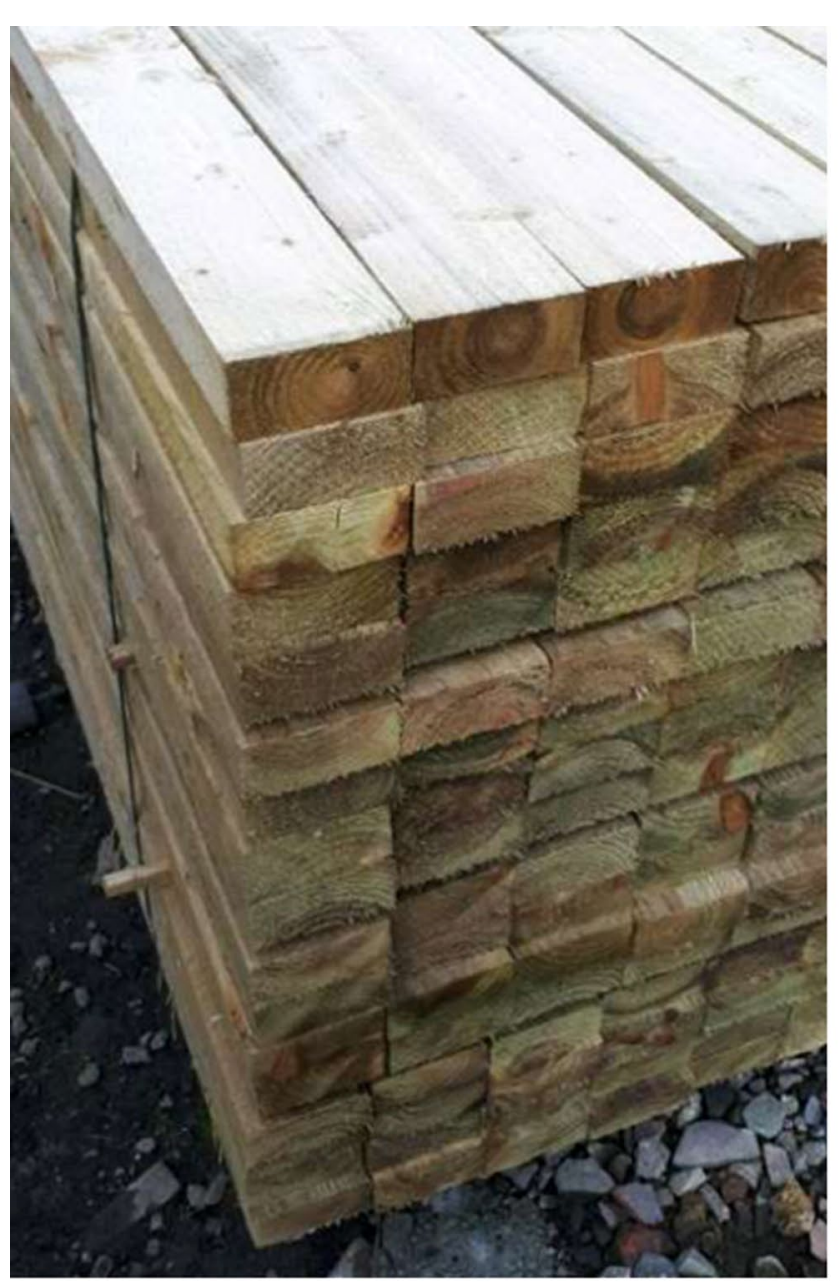

(a)
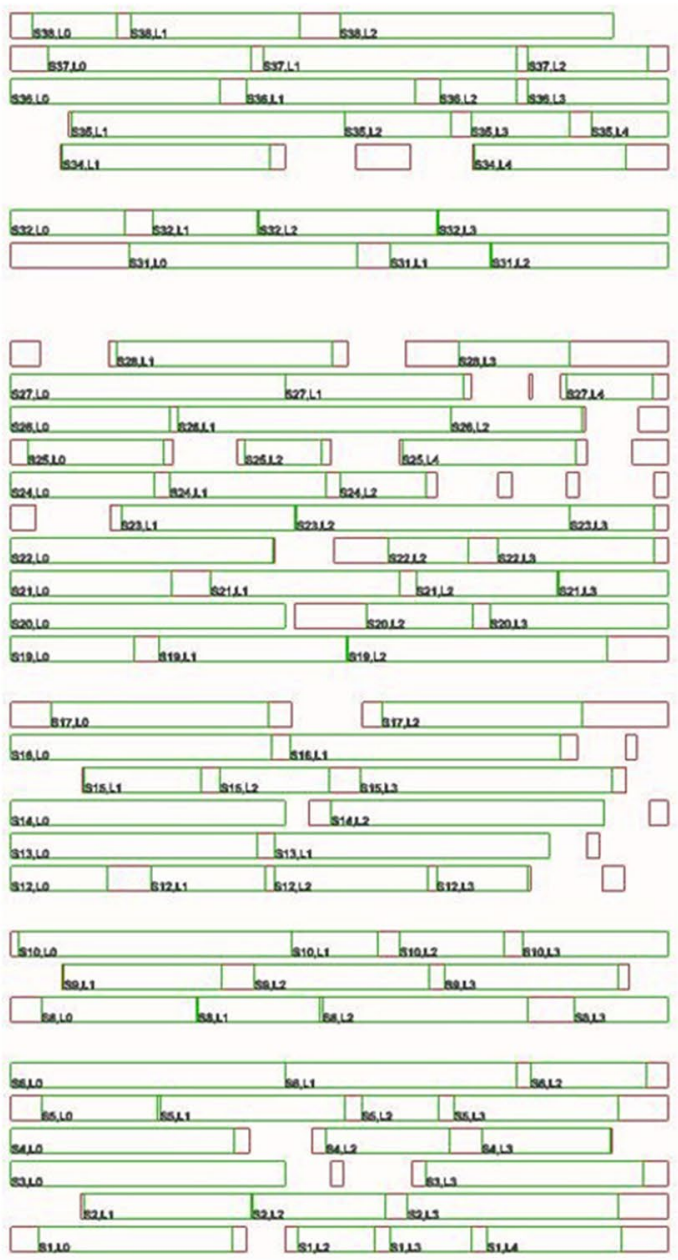

(b)

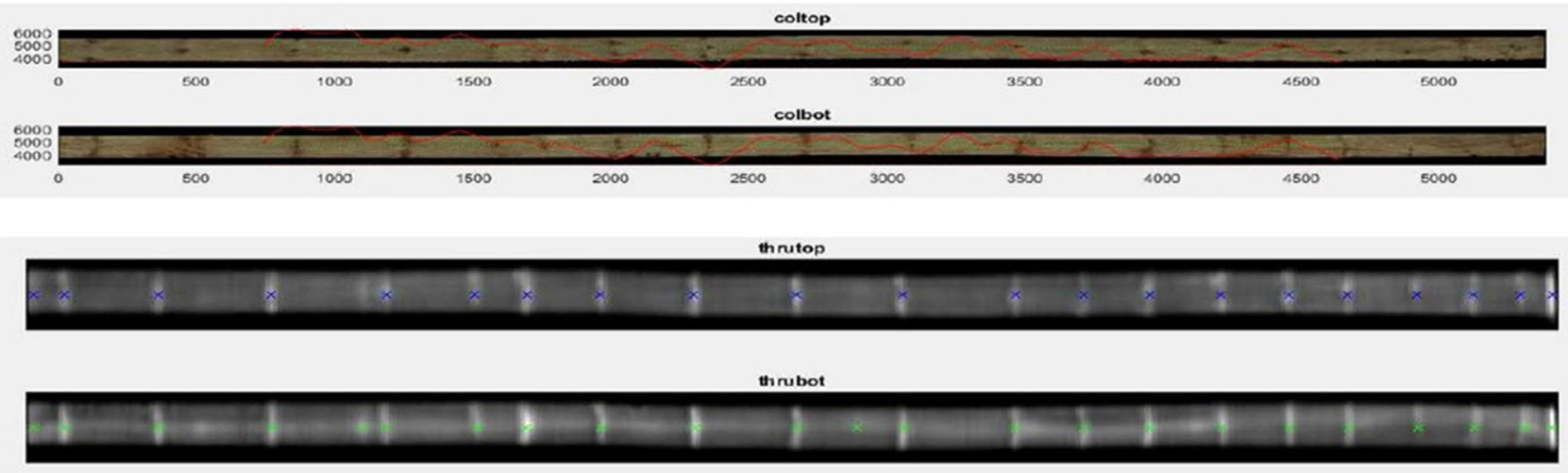

(c)

Fig. 2 a A pack of utility-grade studs containing structural defects $\mathbf{b}$ inventory of usable members after defects are removed c examples of sawmill scan data showing image capture of defects and x-ray scan of density 
of log), knots (penetration from branch growth), warp (bow, spring, or twist within the board), and shakes (cracks or splits within the wood) [2]. For the present project, the preparation of an inventory of short timber lengths was attained through visual inspection for knot-type defects with a size greater than $15 \mathrm{~mm}$, with the resultant inventory of member sizes being the usable lengths after the defects are cut out from the timber.

Additional constraints were introduced for board warp, and a minimum clear length between knots $(280 \mathrm{~mm})$, to ensure practicable fabrication using the process described below in Sect. 4. Parts longer than $1000 \mathrm{~mm}$ were additionally docked to meet the IASS WG21 competition packaging requirements. With an initial stock of 32 utility-grade timber studs, each with a $90 \mathrm{~mm} \times 35 \mathrm{~mm}$ cross section and $2.4 \mathrm{~m}$ length, 120 knot defects were identified with 98 short lengths of usable clear wood between them (Fig. 2).

There are several existing methods proposed to quantify the efficacy of resource recovery in inventory-constrained design processes. A comprehensive life-cycle cost measure can consider material production, fabrication and waste disposal processes, but requires a cost estimation for material processing and waste disposal [16]. A simplified method proposed in $[10,17]$ used an offcut-ratio measure which considers only the total mass of the overall structure and generated waste (unused material). For both measures, there are additional nuances related to whether unused material or offcuts are considered as waste, or considered as available inventory for future use. An additional consideration relevant for the present study is that utility-grade timber is, in its entirety, already considered an unusable waste material generated as a by-product of the structural sawn timber manufacturing process.

Given that the objective of this study is to use as much of the available utility-grade inventory as possible, a simplified 'Resource Recovery' (RR) metric is proposed, where:

$\mathrm{RR}=\mathrm{M}_{\text {structure }} / \mathrm{M}_{\text {material }}$

$M_{\text {structure }}$ is the weight of the members in the final structure and $M_{\text {material }}$ is the weight of the available usable clear wood inventory. The selected 32 studs weighed $109 \mathrm{~kg}$ and the 120 removed knot defects weighed $12.9 \mathrm{~kg}$. The remaining inventory weight from the 98 short lengths of usable clear wood is then $M_{\text {material }}=96.2 \mathrm{~kg}$.

\subsection{Funicular form generation}

A funicular model was developed in response to the design criteria of the "Working Group 21 - Advanced Manufacturing and Materials" exhibition. Given the design area of $4 \times 4 \times 4$ meters, a planar network of generating lines in the ground plan was composed by the design team as shown in Fig. 3a and used as the geometry input for the initial hanging chain simulation. The aim of this was twofold: to maintain the branching network at a valency of three at each node in order to form the three-way joint described in Sect. 4; and to keep the anchor points around the perimeter of the prescribed design boundary, so that the pavilion would maximise its footprint within the defined space.

The spacing and distribution of the anchors were intended to create a desired internal spatial composition, with the anchor points at the perimeter of the design boundary being densely arrayed such that the resultant arches would form a clear delineation between the interior and exterior spaces once erected. Two pairs of adjacent anchors were kept at a wider distance to form a clear entry
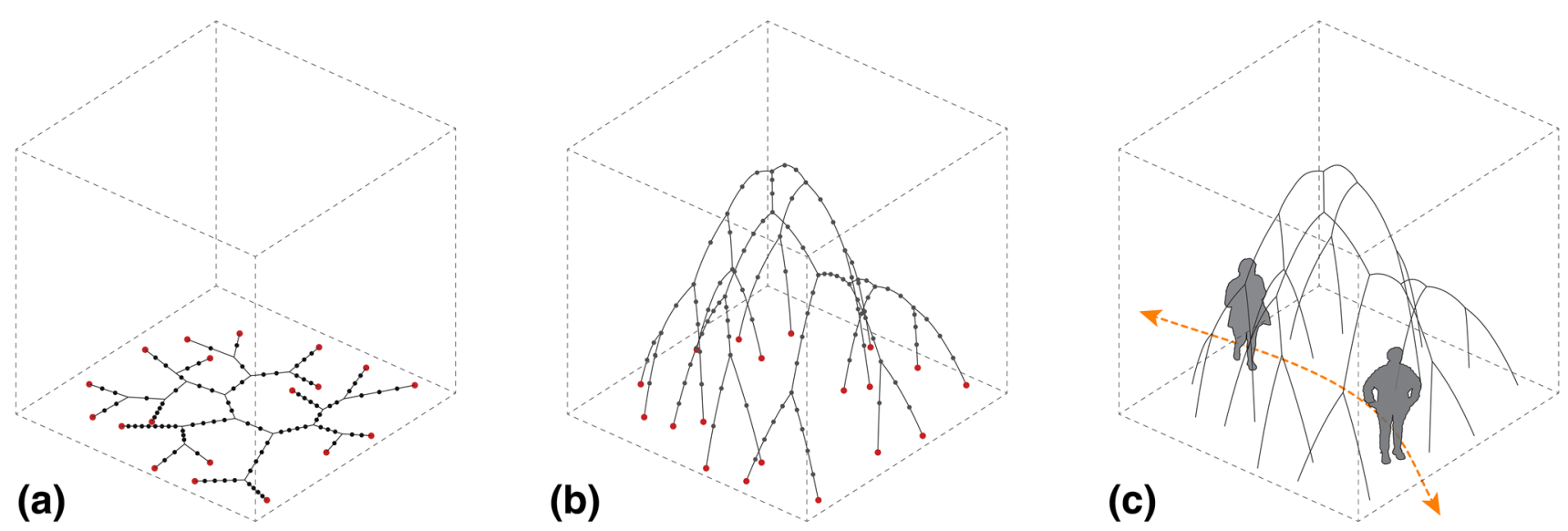

Fig. 3 Funicular form generation: $\mathbf{a}$ the initial planar network of generating lines with subdivisions; $\mathbf{b}$ relaxed geometry in the hanging chain simulation; c resultant pavilion form with smoothed arches and circulation path shown 
and exit and circulation path through the pavilion in the exhibition (Fig. 3c).

The generated lines within the initial planar network were further subdivided into a series of shorter lines that were used to derive the relaxed springs in the hanging chain model simulation. An equal force emulating the reversed gravity was then applied to each node of the spring network in the virtual model. The number of subdivisions applied to each of the intitially generated lines could also be independently varied to provide manipulation of the final length and curvature for each resultant hanging chain, allowing fine-tuning of the overall form.

In this initial relaxation simulation, the number of subdivisions per line was set to fulfil two primary criteria: 1 : To ensure that the overall relaxed hanging chain model was set with a height limitation of four metres, with sufficient internal head height clearance for circulation when it reaches the equilibrated state; and 2: To ensure that the chains of springs in the simulated model did not result in arches with excessive distortions in localized curvature. At this stage of the simulation, the number of the subdivisions of the initial line network was used for formal composition rather than correlating with the given inventory of short length timbers.

\section{Part assignment}

\subsection{Part assignment heuristic}

In considering an inventory assignment method, a key limitation was identified in existing research: inventories and target structural forms are typically assumed to both consist of straight elements. Assignment algorithms for curved inventory pieces have had relatively limited exploration, although the notable example of the Biomass Boiler House [18] used naturally curved trees to populate a long, curved building wall. The present study seeks to assign straight (short length timber) parts to curved (funicular chain) members, proposing a best-fit heuristic that includes three novel components: curved part generation from straight inventory pieces; an all-targets best-fit assignment based on tangent difference; and funicular form regeneration through enforced G0 (curve end position) or G1 (curve end tangent) continuity.

First, a strategy to machine curved inventory segments from straight stock material was adapted based on the technique utilized by the authors in [19]. With reference to Fig. $4 a$, the sagitta distance is used as a control parameter to nest a maximally-sized curved inventory part inside the initially-straight timber length. When a uniform sagitta is applied to straight segments of varying length, a new inventory is established with parts of varying radii of curvature, with shorter lengths generating tighter curvatures.

Second, a best-fit heuristic approach was selected as appropriate for part assignment in the present context. The relatively high speed of a heuristic approach enables rapid designer feedback as to the amount of inventory use and the quality of assignment [6]. With reference to Fig. $4 \mathrm{~b}$, 'best' was defined as the smallest tangent difference, measured as the angle between the end tangent of an inventory element and a tangent evaluated at a point on the target curve at a matching arc length. The tangent difference was evaluated between all inventory parts and all targets, and part assignment proceeded in order of inventory elements with the smallest tangent difference. After each iteration, the best-fit inventory element was removed from available parts, the best-fit target curve was truncated at assignment length, and the remaining target curve was updated in the list of targets with tangent difference re-evaluated. The assignment algorithm terminated when all inventory parts were used, or when all remaining inventory parts were longer than all the remaining length of the target elements.

Third, inventory parts were reassembled into an approximation of the target funicular curve. If inventory parts are assembled assuming end points aligned to the target 
(a)

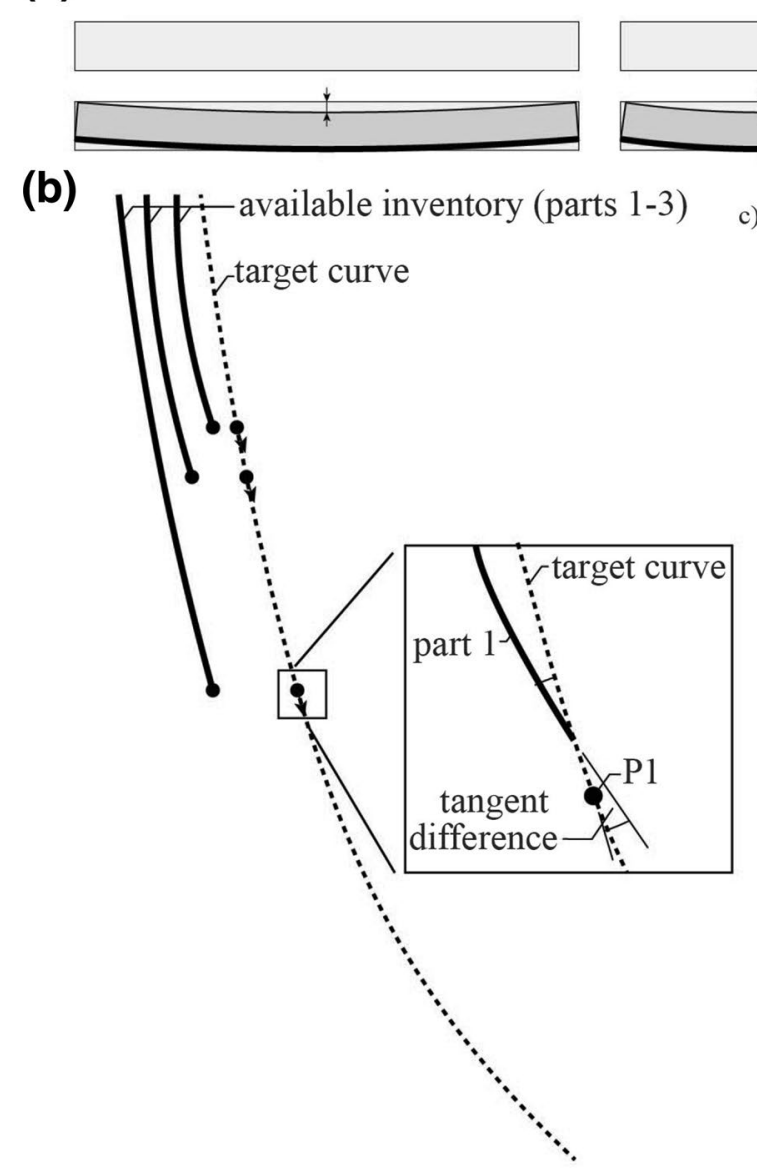

Fig. 4 Curved inventory generation and assignment. a Straight stock to curved inventory conversion. b Best-fit heuristic with evaluation of tangent difference between inventory and target curves.

curve, the assembly has G0 (curve end position) continuity. Alternately, inventory parts can be considered as piecewise arcs and assembled with aligned end tangents and enforced $\mathrm{G} 1$ continuity. Both strategies are shown in Fig. 4c, with the $\mathrm{G} 1$ continuous curve constructed using the generative grammar proposed in [20]. Good fidelity is seen in both cases, where fidelity is measured as the cumulative nearest point distance between the assembled inventory and target curves. Preliminary benchmark testing of part-assignment for a single funicular cable showed that the $\mathrm{G} 1$ assembler had better fidelity when selecting from a large inventory of parts, and worse fidelity when
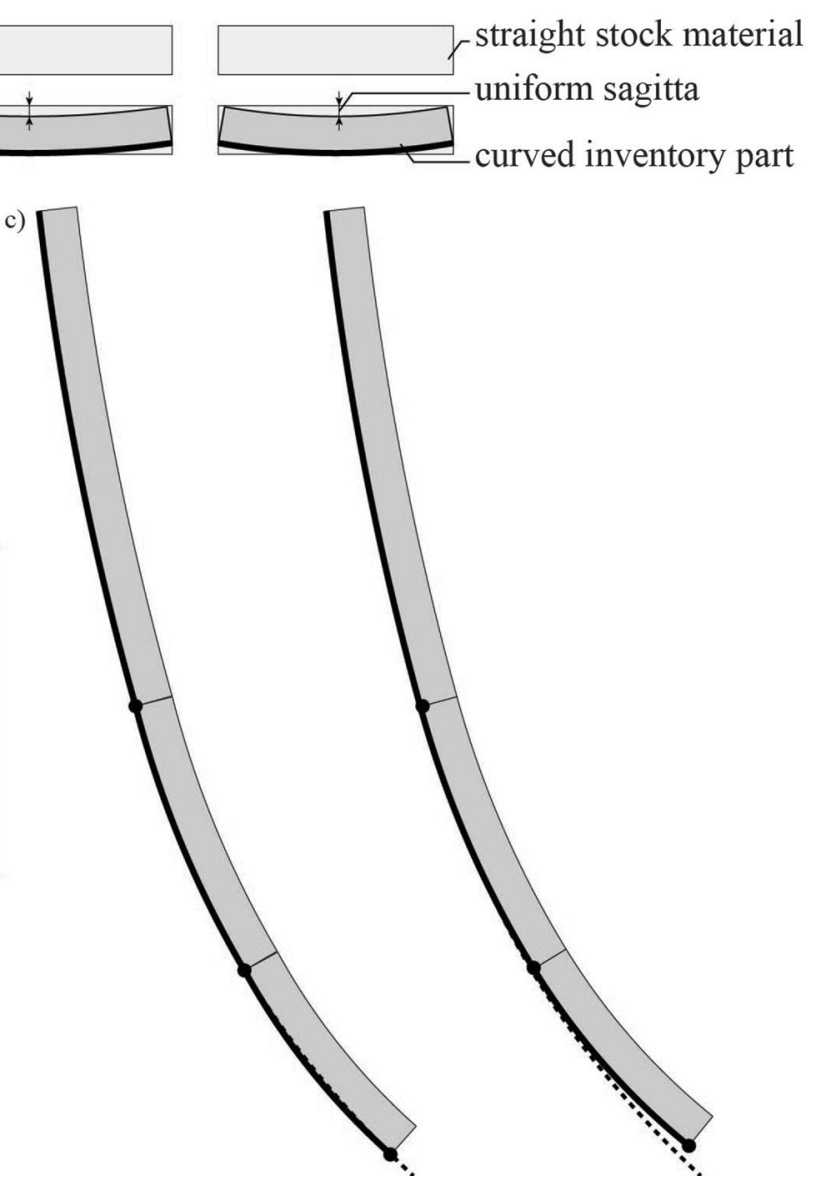

$\mathrm{P} 1$ is a point on the target curve evaluated at the arc length of part 1. c Inventory parts assembled with G0 (left) or G1 (right) continuity

selecting from a constrained (minimum number required) inventory of parts.

\subsection{Evaluation and re-relaxation}

The part assignment heuristic was tested on the funicular form described in Sect. 2, using the 98 inventory pieces with lengths varying from 280 to $1000 \mathrm{~mm}$. Figure 5 shows the part assignments at 5, 20, and 81 iterations, mapped onto the 27 target curves comprising the funicular model. The algorithm completed in a fast $3.5 \mathrm{~s}$ (Intel i7-4790 3.6 GHz CPU, 16 Gb RAM, SSD) at 81 inventory 


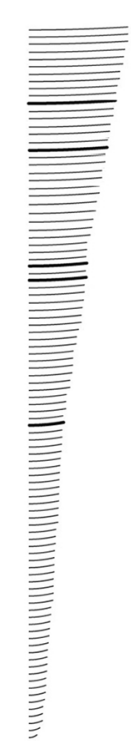

(a)

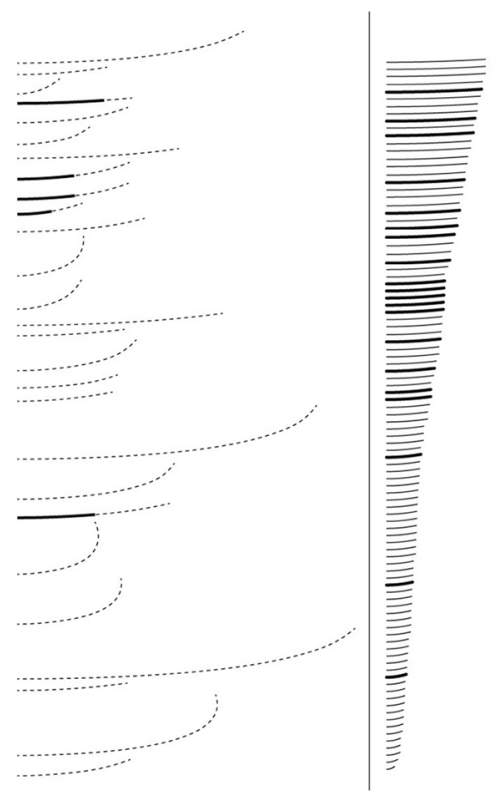

(b)

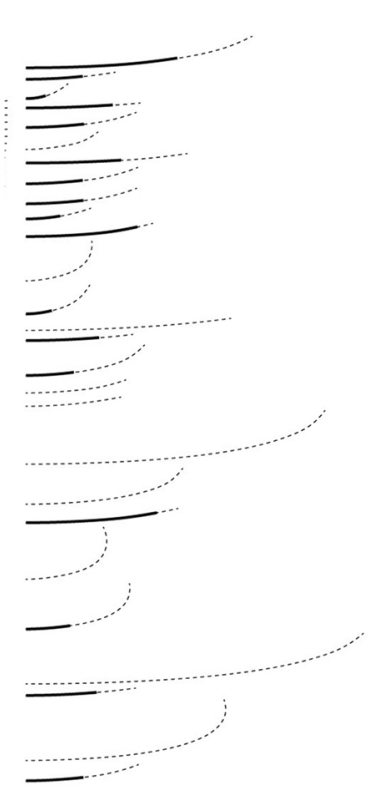

(c)

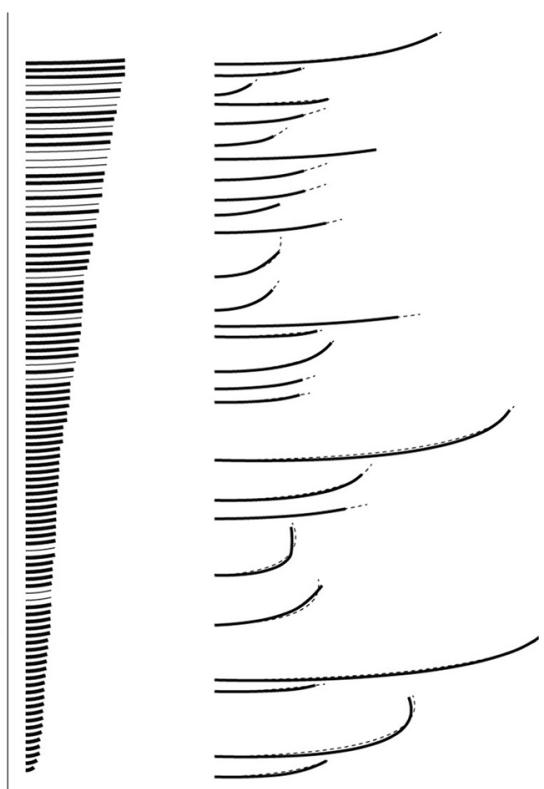

Fig. 5 Part assignment results with a 5, b 20, and c 81 (final) parts. Target curves shown as dashed lines, unassigned inventory parts shown as thin lines, assigned inventory parts shown as thick lines

assignments, with termination due to no remaining shorter-length inventory. Observation of assignment ordering shows that longer and less-curved parts are assigned early and are well matched with the initial, lowcurvature regions of the funicular cables. Shorter and more tightly curved parts are preserved for later iterations, which again suits the increasing funicular curvature and decreasing remaining target lengths. The use of minimum tangent difference ordering criteria is concluded to be a rational choice of pre-sorting criterion and results in good quality solutions for this problem.

Based on the results of the part assignment heuristic, the selected inventory items are assembled with G1 continuity to approximately form the target curves of the funicular form. The reconstructed curves are then mapped 


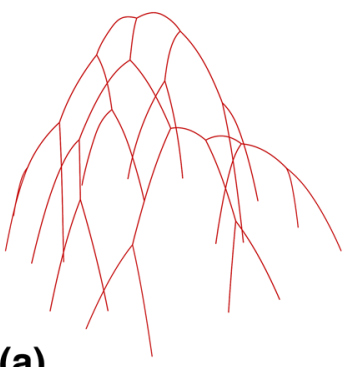

(a)

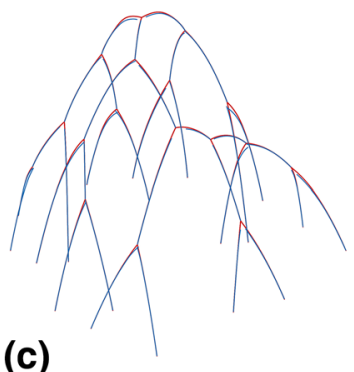

(c)

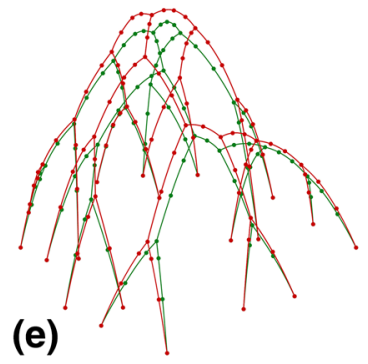

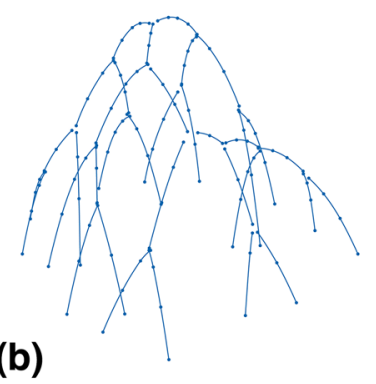

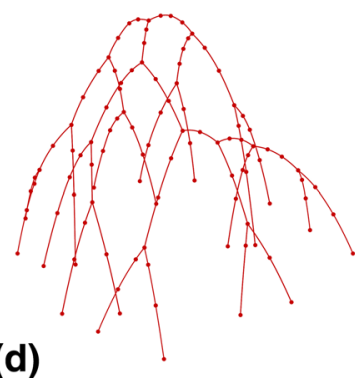

(d)

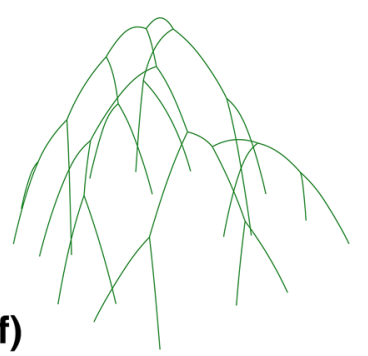

Fig. 6 Second round relaxation process: a initial relaxation $\mathbf{b}$ arch segments re-assembled based on part assignment results $\mathbf{c}$ overlay and comparison between $\mathbf{a}$ and $\mathbf{b}$; $\mathbf{d}$ second round re-subdivision of chains now based on the number of inventory parts assigned in the heuristic; e re-relaxation of the hanging chain to meet target lengths of assigned parts, overlaid on $\mathbf{d}$; $\mathbf{f}$ result from second round relaxation

to their original locations, as shown in Fig. 6 a-c. A second stage relaxation was then executed to update the initial funicular form with the additional information acquired through the part assignment heuristic.

As depicted in Fig. 6d, each chain in the original funicular form is re-subdivided in the new simulation; such that for each chain, the number of subdivisions, and therefore resultant springs is set to match the number of assigned parts. In Fig. 6e the target length of each spring is then set to also match the length of the corresponding inventory part. In this second-round simulation, the target length criterion is also given the utmost priority as compared to other input simulation factors such as the gravitational load and the preservation of the locations of footing anchor points.

In comparing the original and re-relaxed forms, it is evident that the second round simulation process caused some localized undesirable adjustment to chain curvature, as it matched target part lengths whilst maintaining the ground anchor point boundary conditions. Figure $7 \mathrm{a}$ shows this case, where the ground anchor positions were strictly constrained to their original location, and a local connecting chain was subject to a significant change in curvature. Consequently, the segments in the resultant arch were significantly distorted compared with their originally assigned inventory parts, as shown in Fig. 7b. An improved hanging chain simulation strategy was therefore developed for the second round relaxation, as shown in Fig. 8a, where the relative magnitude of the ground anchor constraint is reduced such that the locations of the anchors are allowed to adjust relative to their initial location. This additional interactive fine tuning of the hanging chain simulation results in a closer correlation to the original curvature, as shown in detail in Fig. 8b. 
Fig. 7 Simulation case where ground anchor point locations are locked during re-relaxation: original relaxed geom-

etry shown in red, re-relaxed geometry shown in green. a $3 \mathbf{d}$ and plan view $\mathbf{b}$ detailed elevation view of localised chain curvature undergoing significant distortion relative to the assigned inventory parts
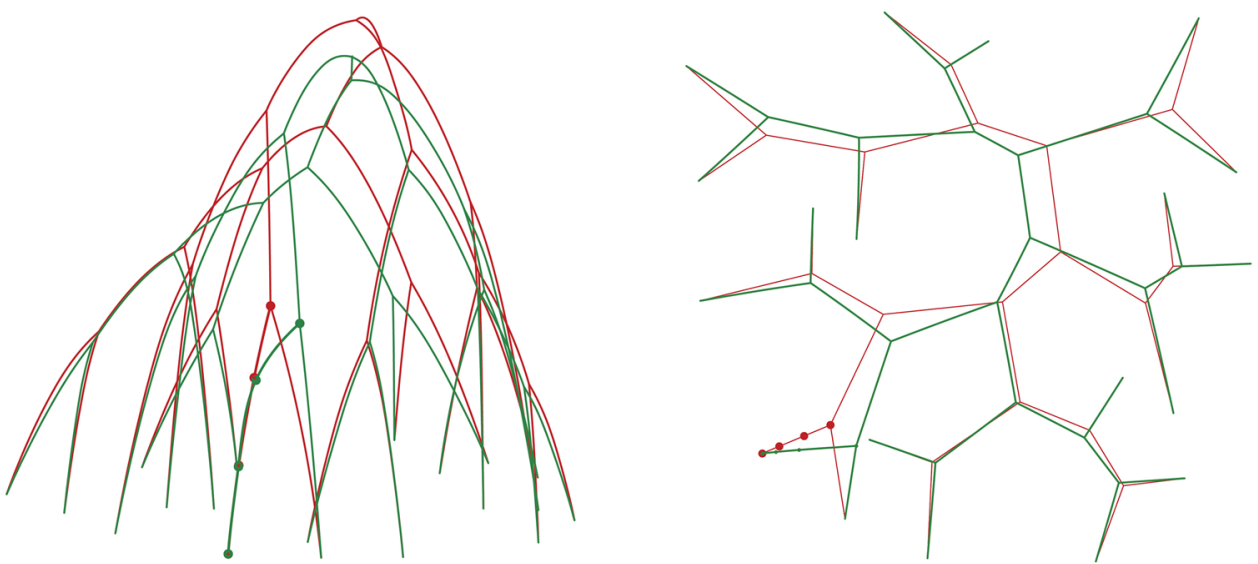

(a)
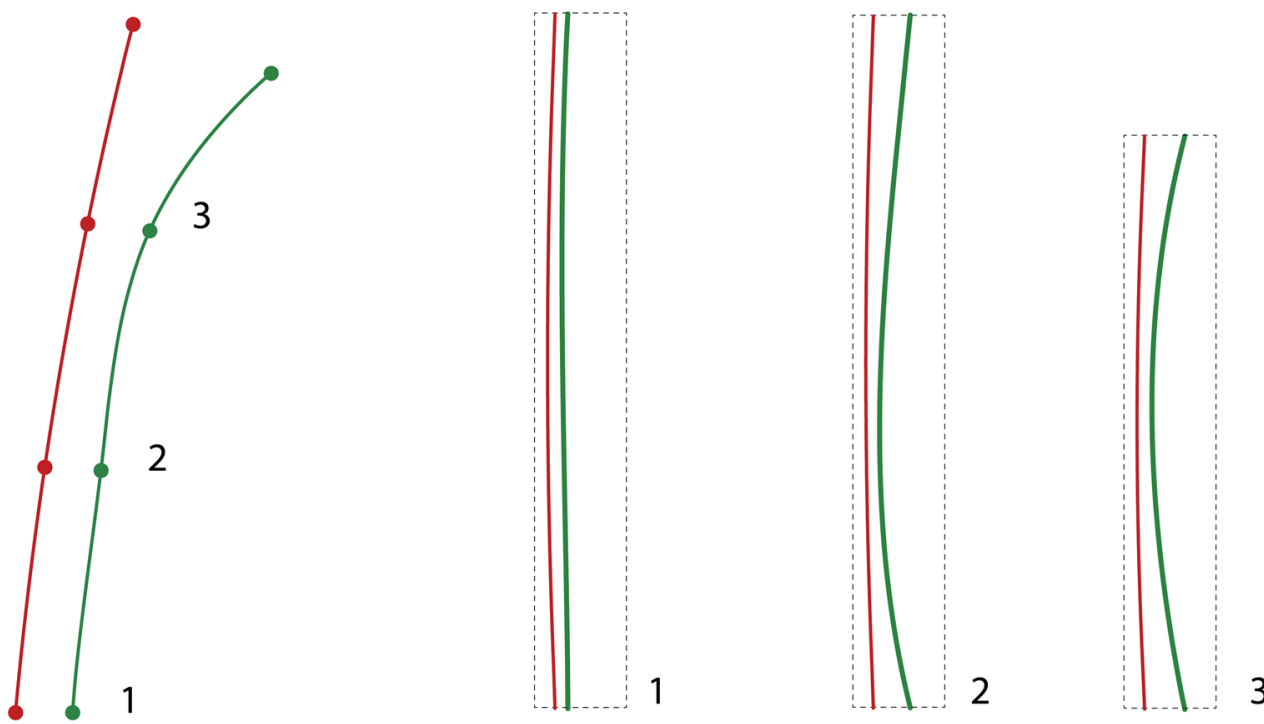

(b) 
Fig. 8 Simulation case where ground anchor point locations are permitted to adjust during re-relaxation: original relaxed geometry shown in red, re-relaxed geometry shown in green. a $3 \mathrm{~d}$ and plan view $\mathbf{b}$ detailed elevation view of localised chain curvature correlating to the assigned inventory parts
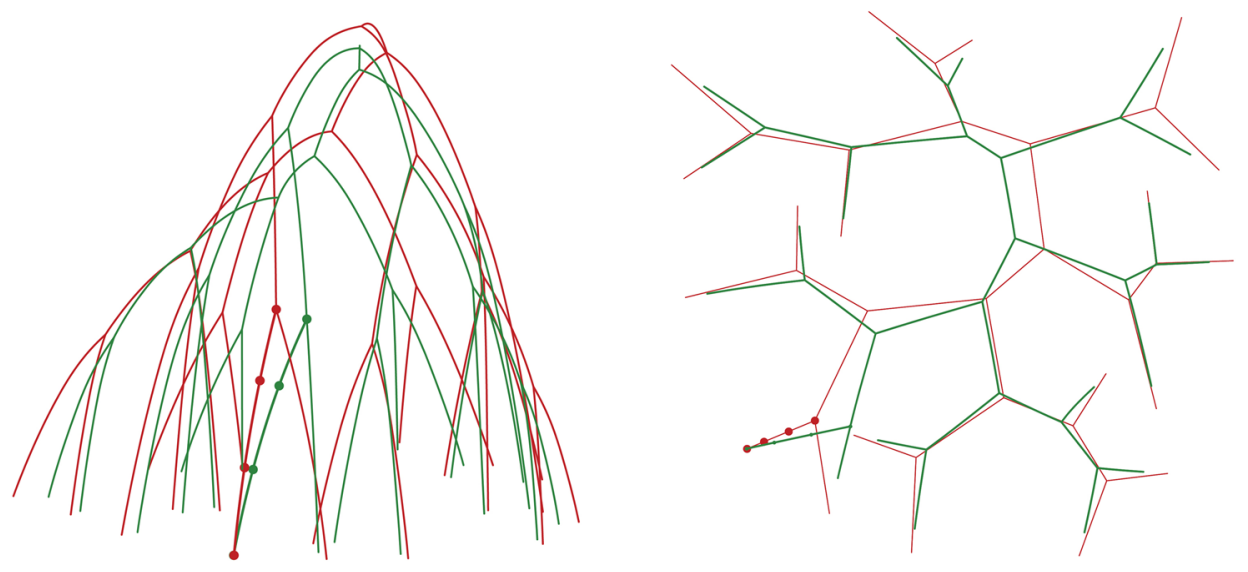

(a)

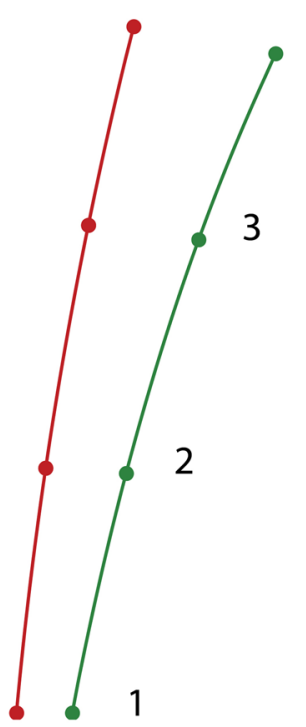

(b)
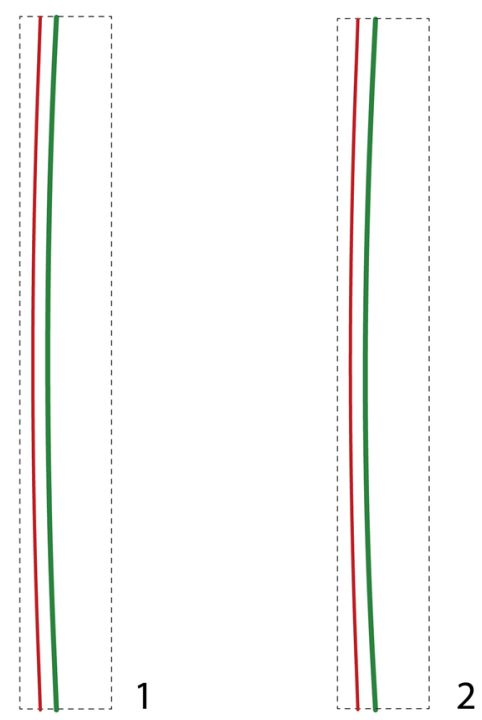

\section{Timber fabrication}

Upon finalizing the geometry of the re-relaxed funicular model, the centreline paths were used to generate solid geometry for fabrication. Connection design was a key consideration in part generation, given the IASS WG21 competition requirement for the structure to be re-deployable, two connection designs were required to be resolved: the two-way end to end connection of coplanar members; and the three-way bifurcated connection of non-co-planar members. Both connections required a degree of semi-rigidity in order to provide overall stiffness to the structure, both in its completed form and during assembly. 


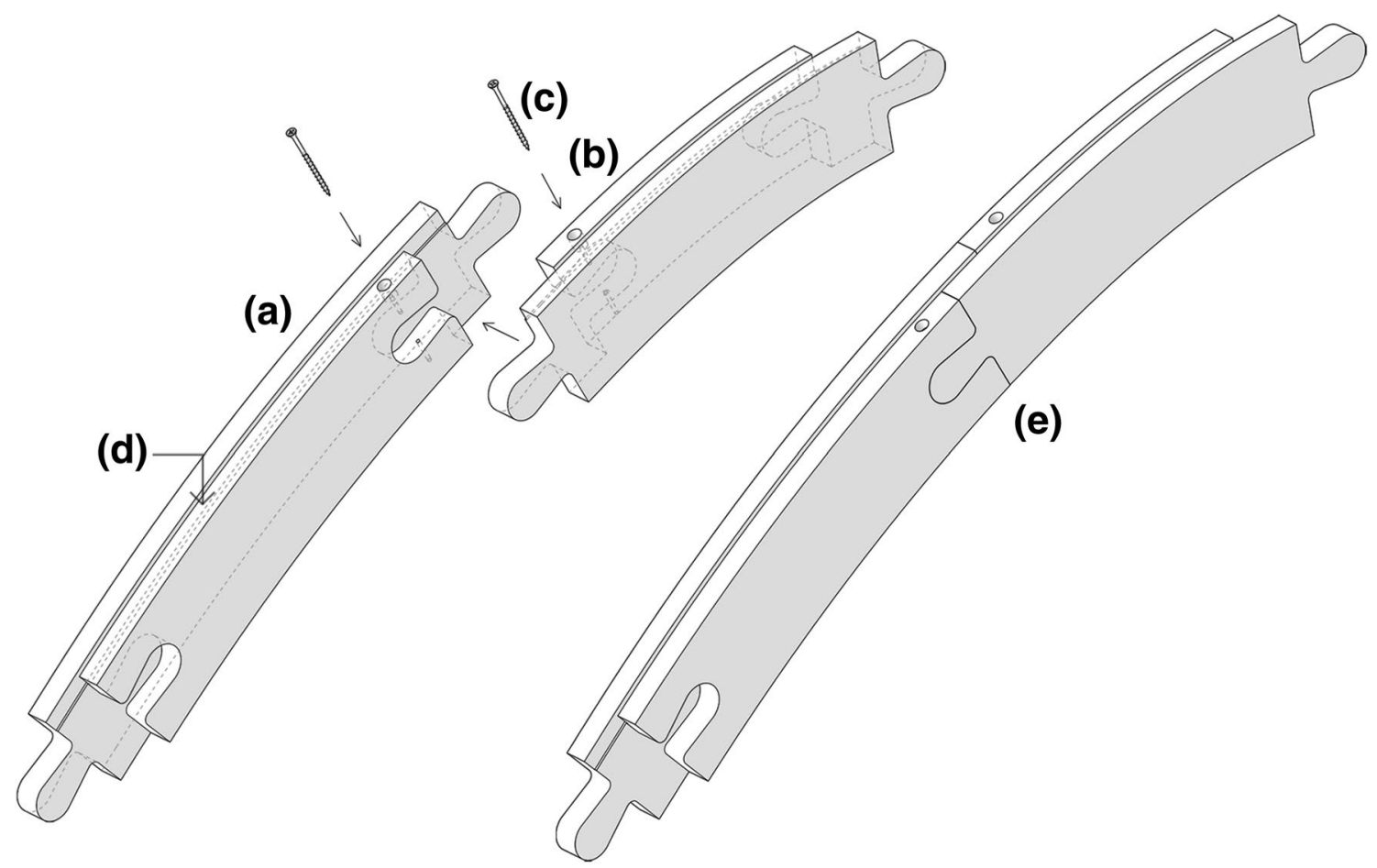

Fig. 9 Two-way lapped dovetail joint assembly: a, b arch segments; c counterbored screws—subsequently concealed behind fabric skin; $\mathbf{d}$ rebate for fabric skin insertion; e assembled joint

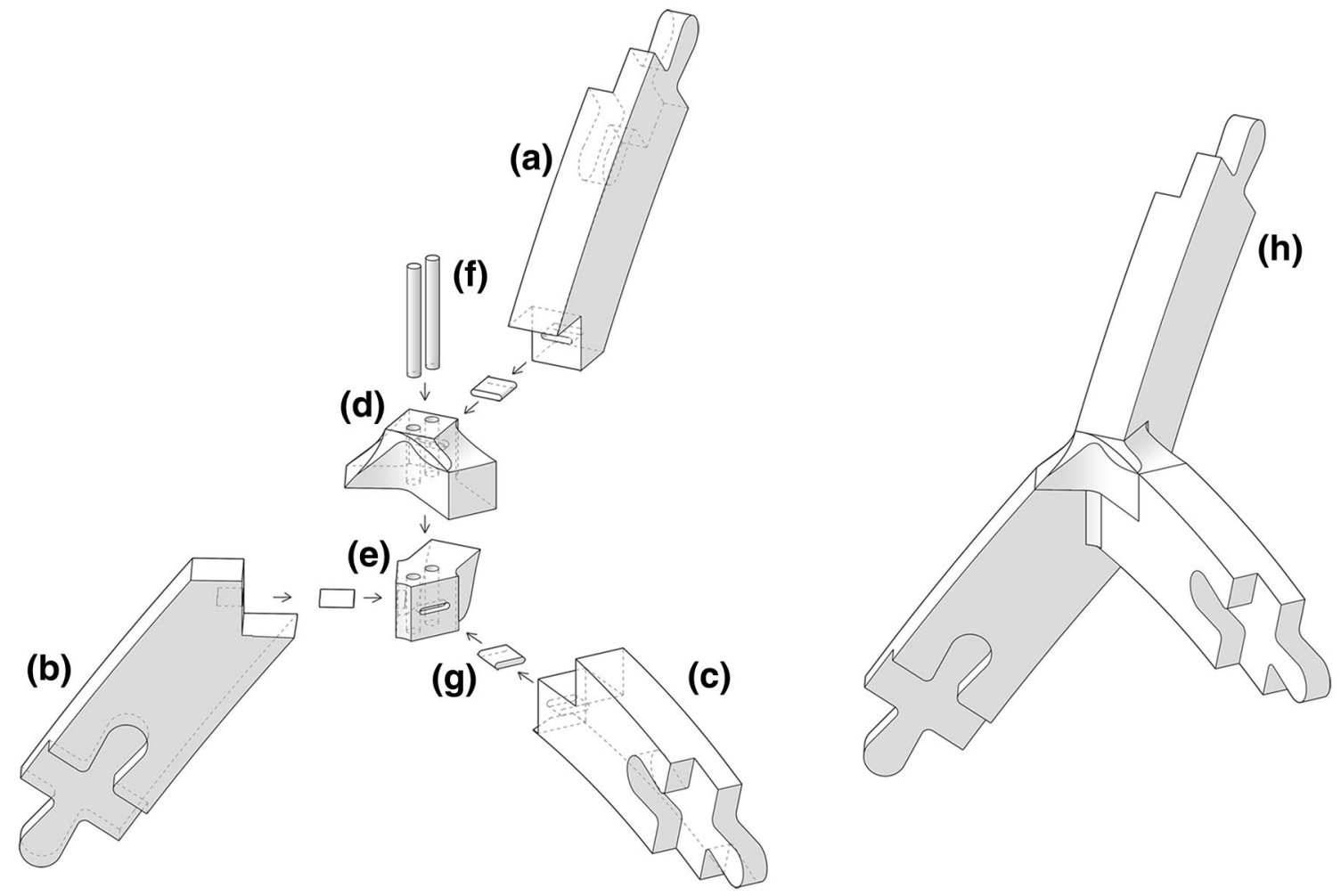

Fig. 10 Three-way joint assembly: a-c 'node tail' elements that connect to adjacent arches; $\mathbf{d}$, e coupled node blocks joined with; $\mathbf{f}$ timber dowels; connecting to the 'node tails' via $\mathbf{g}$ timber dominoes; $\mathbf{h}$ assembled joint

\section{SN Applied Sciences}


(a)

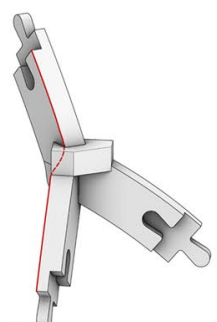

(b)
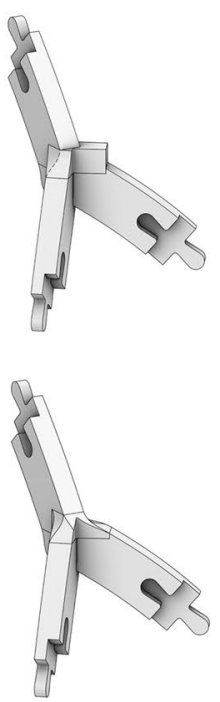

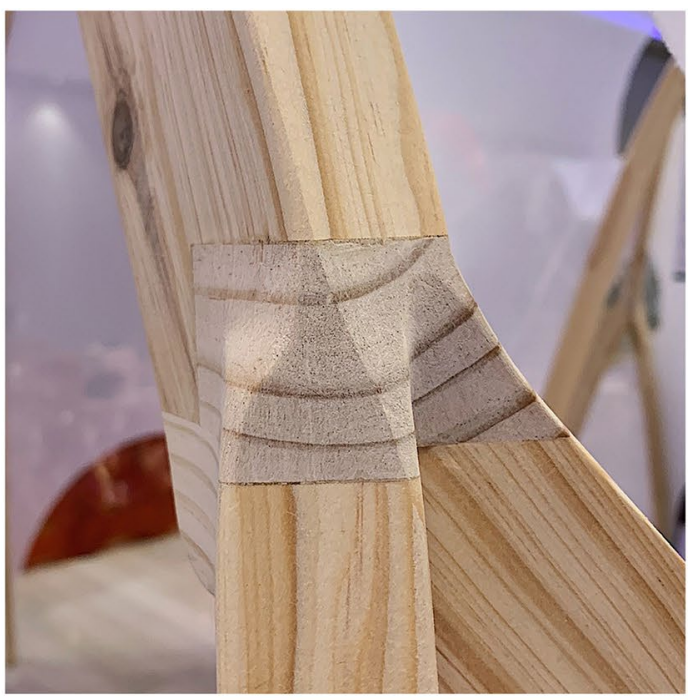

(c)

Fig. 11 Node block trimming: $\mathbf{a}$, $\mathbf{b}$ swept spline surfaces transition between the tangents of each of the leading arch edges, used to trim the solid geometry; c assembled node

Fig. 12 Machining of a typical arch segments with two-way connection, $\mathbf{b}$ three-way nodeblock components $\mathbf{c}$ un-assembled node-block components and node tails

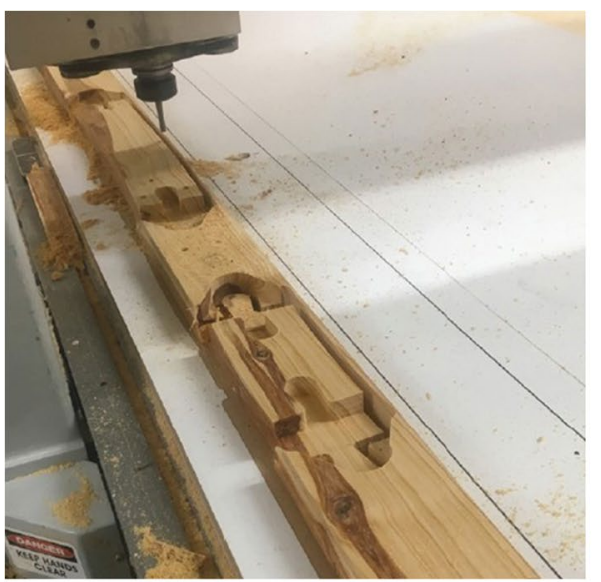

(a)

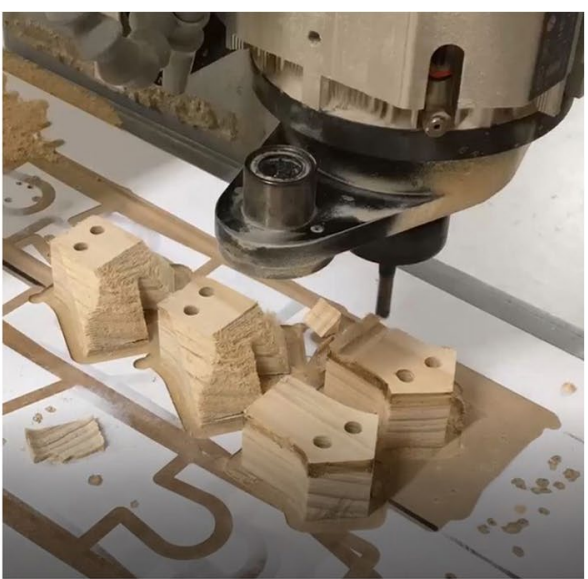

(b)

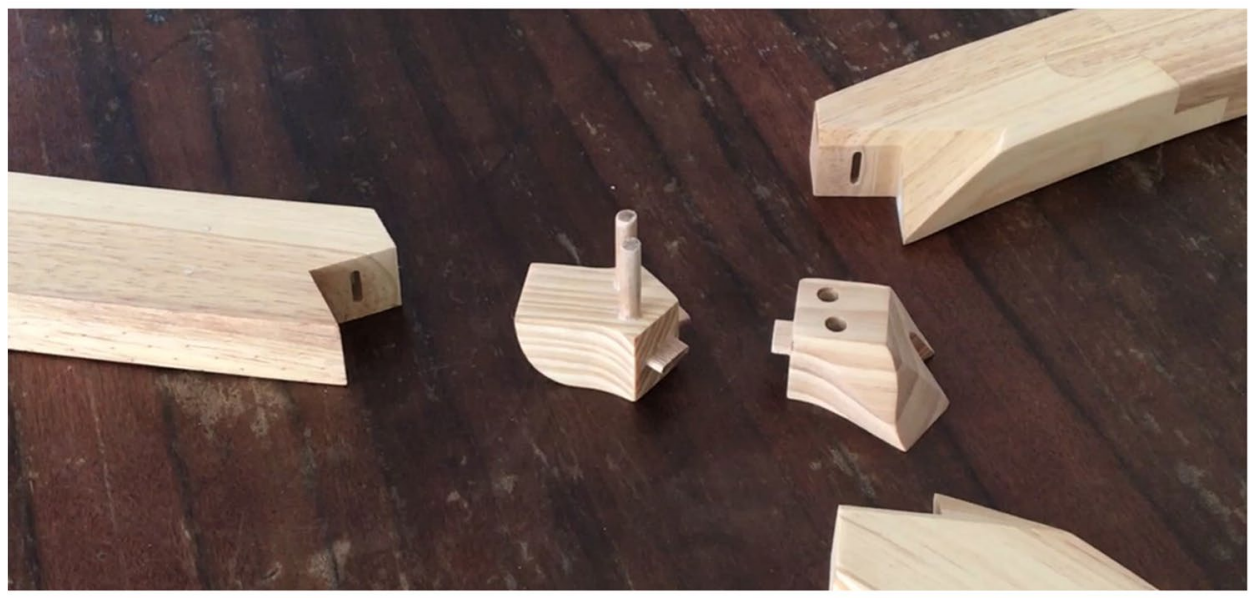

(c) 


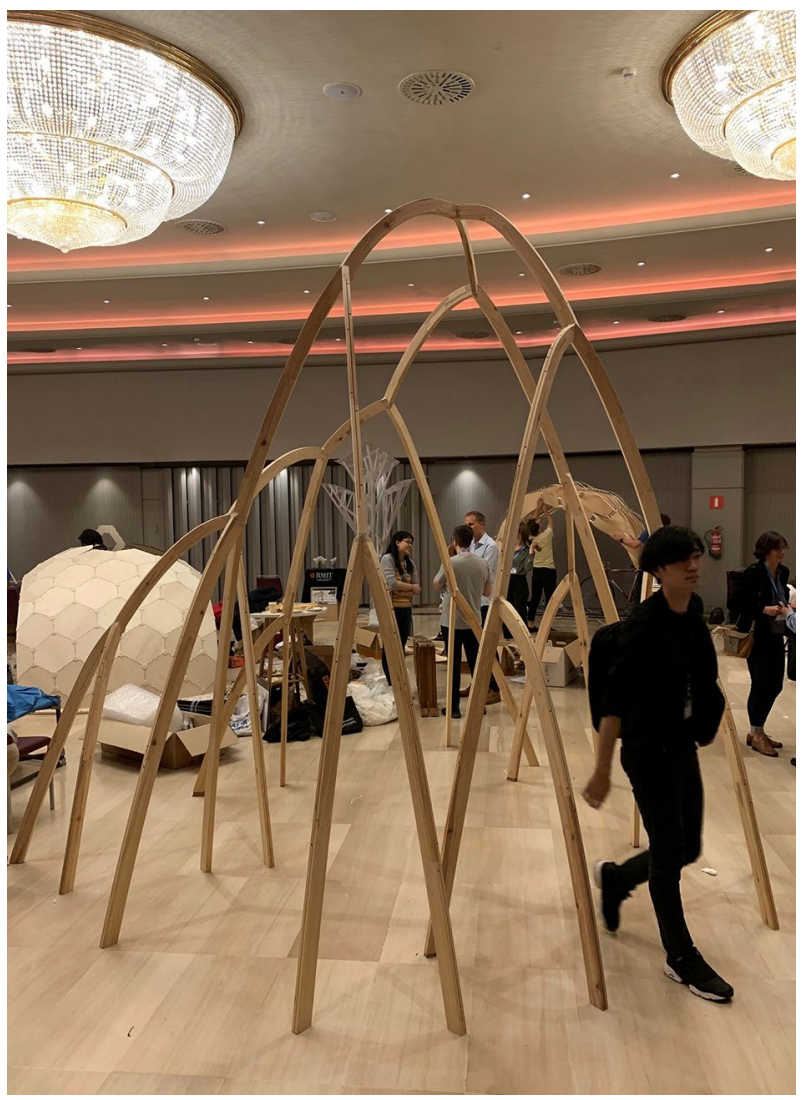

Fig. 13 Assembled timber structure, by authors

For the two-way joint, the solution pursued for achieving semi-rigidity was a tight fitting lapped dovetail joint that relied on calibrated machining tolerance $(0.1 \mathrm{~mm})$ to provide an effective timber to timber mechanical interlock, allowing only a single degree of freedom in the direction of insertion. During assembly, this was additionally fixed with two counterbored screws, to account for any dimensional change and joint loosening which may occur from transport or repeated assembly. The connection is shown in Fig. 9.

For the three-way joint, the principal design criterion was resolving the intersection of the non-coplanar members meeting at a node with varying angles of incidence. A constraint imposed upon the design of this connection was that it be machinable on a CNC router with only 3 axes, which would enable this type of connection to be readily fabricated in modestly equipped workshops out of inexpensive timber. Toolpath constraints in 3 axis router production prevent any undercutting of material and this became a primary driver for the development of a 'coupled' node, consisting of a pair of timber blocks of regular framing timber (cut from the same sized timber stock as the arched members), coupled together to form a central node block. These blocks are designed to be contour routed from the top-down, with one of the blocks flipped upside down to form the bottom half. Each of the intersecting arches that meet at the node connect with a short arched segment, or 'node tail', connected to the node via a rabbet joint. The overall node is therefore constructed from 5 elements, as shown in Fig. 10.

The outer surfaces of the two node blocks were trimmed to provide smooth transitions between connecting arches. A series of splines were created by connecting the tangents of the leading edges of the incoming arches, and used to generate sweep trim surfaces that bridge the flat surfaces of the joining arches as shown in Fig. 11. Only singly-curved surfaces were used in this design process, allowing the node to be readily polished using a linisher.

Fabricated components for both node types are shown in Fig. 12. The three-way joint included timber dowels and dominoes for component alignment and to provide additional structural shear capacity. The 5 elements making up the three-way joint were glued together as a transportable pre-assembled element, facilitating rapid and intuitive on-site connection to its adjacent arch segments. The complete timber structure is shown in Fig. 13.

\section{Fabric skin}

A translucent fabric was introduced to define the interior and exterior spaces and the entry points of the pavilion. The skin was conceived as a continuous tensile membrane sleeve with seam lines following arch ridges, shown in Fig. 14. The membrane would also be attached to the structure along these arch ridges, using a concealed rebate detail shown in Fig. 14a-c. 

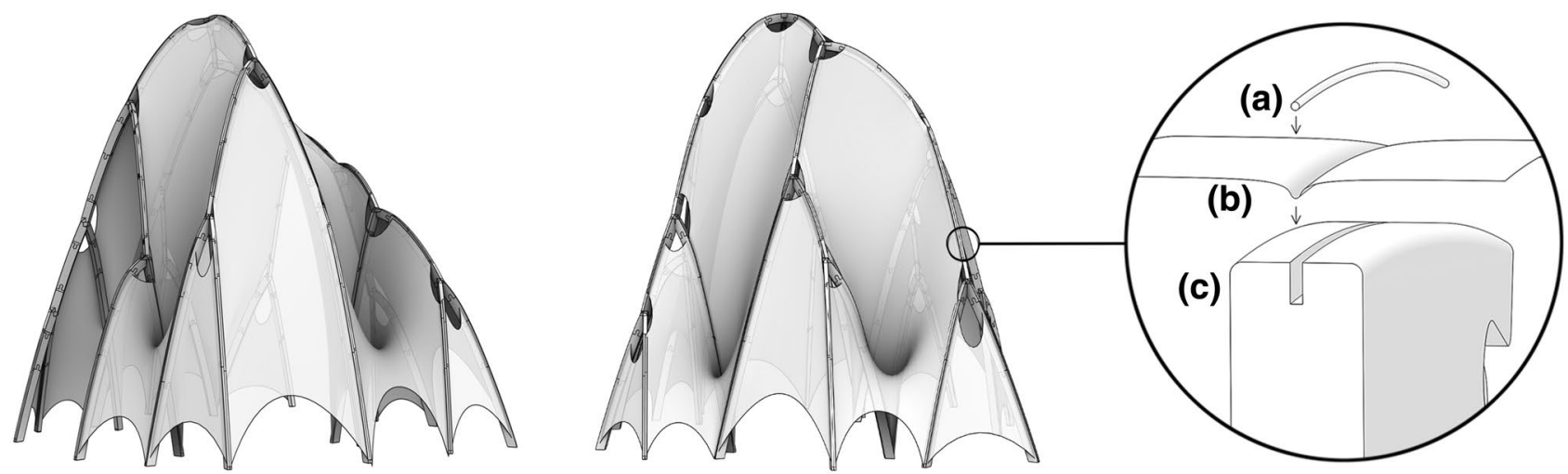

Fig. 14 Geometry of stretched fabric and detail of fabric attachment: a $3 \mathrm{~mm}$ nylon cord that presses b the fabric with sewn seam into c the timber arch with a continuous machined rebate

Additional surface details were introduced to emphasise aspects of the timber structure. Voids in the fabric were deliberately created at nodes to reveal and highlight the three-way joint. The skin was also stretched and attached to the internal arches at selected footing locations to form complex funnels, which can be read as vaults from the interior space.

Fabric modelling and pattern generation was achieved via digital processes. Fabric segments were simulated as minimal surface membranes using dynamic relaxation. These were geometrically discretized into developable polyhedral strips that approximated the minimal surface [21-23]. For the final design, the minimal surface was first discretised into a low-resolution triangular mesh with a limited number of elements, sufficient to maintain a reasonable geometric correspondence but reducing the number of potential strips for sewing. To reduce unnecessary seam lines and maintain visual simplicity, strips at locations with low curvature change were grouped into large pieces. Strips in the funnel area remained separate due to excessive local curvature. A generated projection of a typical intra-arch fabric segment is shown in Fig. 15a and details of the funnels shown in Fig. 15b-c.
Developable strips were translated to two-dimensional cutting templates for materialising in a highly elastic, 2-way stretching 'Power Mesh' knitted nylon fabric, determined through prototyping to have approximately a $20 \%$ stretch rate. The final cut template was thus scaled to $80 \%$ to purposely introduce tension to the fabric and avoid potential wrinkling, a method used in the membrane fabrication industry [21].

Finally, all cut templates were nested and laid out on a $1500 \mathrm{~mm}$ wide fabric roll, with $140 \mathrm{~mm}$ grid spacing as shown in Fig. 16. These were cut by hand and machine sewn with a zigzag stich, with the fabric stretch allowance aiding in mitigating a considerable amount of infidelity/inaccuracy in the cut template translation process.

The fully-sewn fabric was stretched over the timber structure, with fabric seams secured and concealed in the continuous rebates, and anchoring fixings at the bases of arches to maintain tension throughout the fabric. The completed pavilion, as exhibited at the IASS conference in Barcelona from the 6th-1 $11^{\text {th }}$ October 2019 is shown in Fig. 17. 


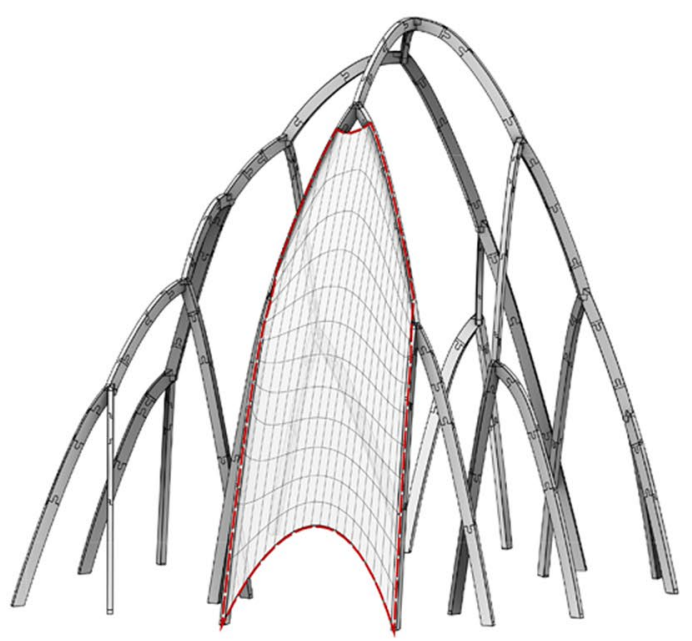

(a)

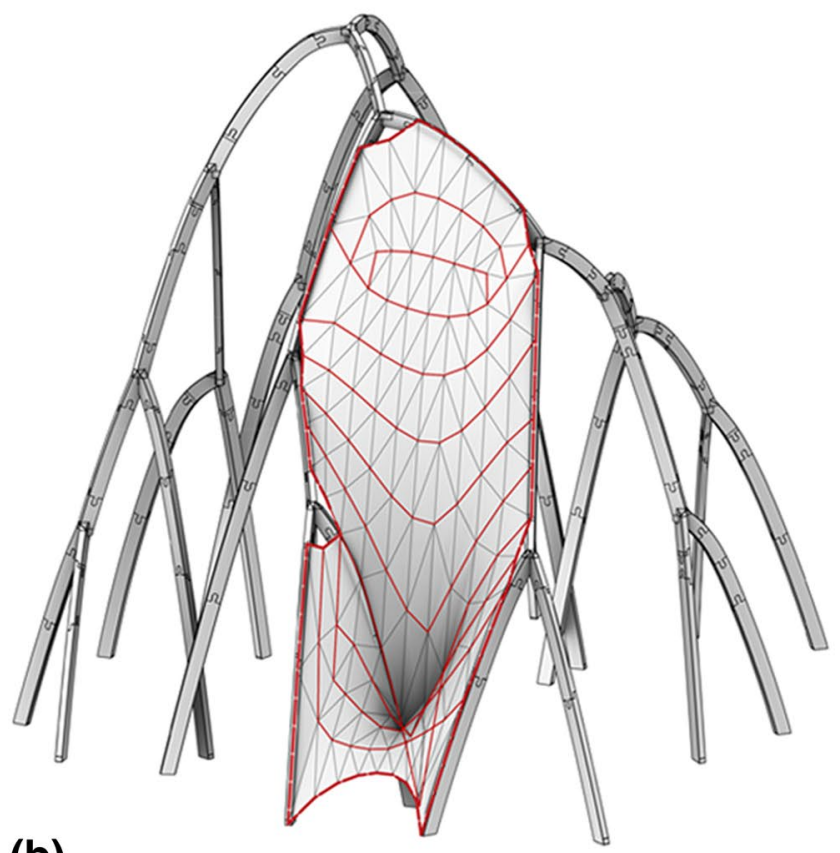

(b)

Fig. 15 a Selected simple segment of fabric being projected to create an outline and subsequently scaled down by $20 \%$ for the cutting template b selected complex segment approximated with
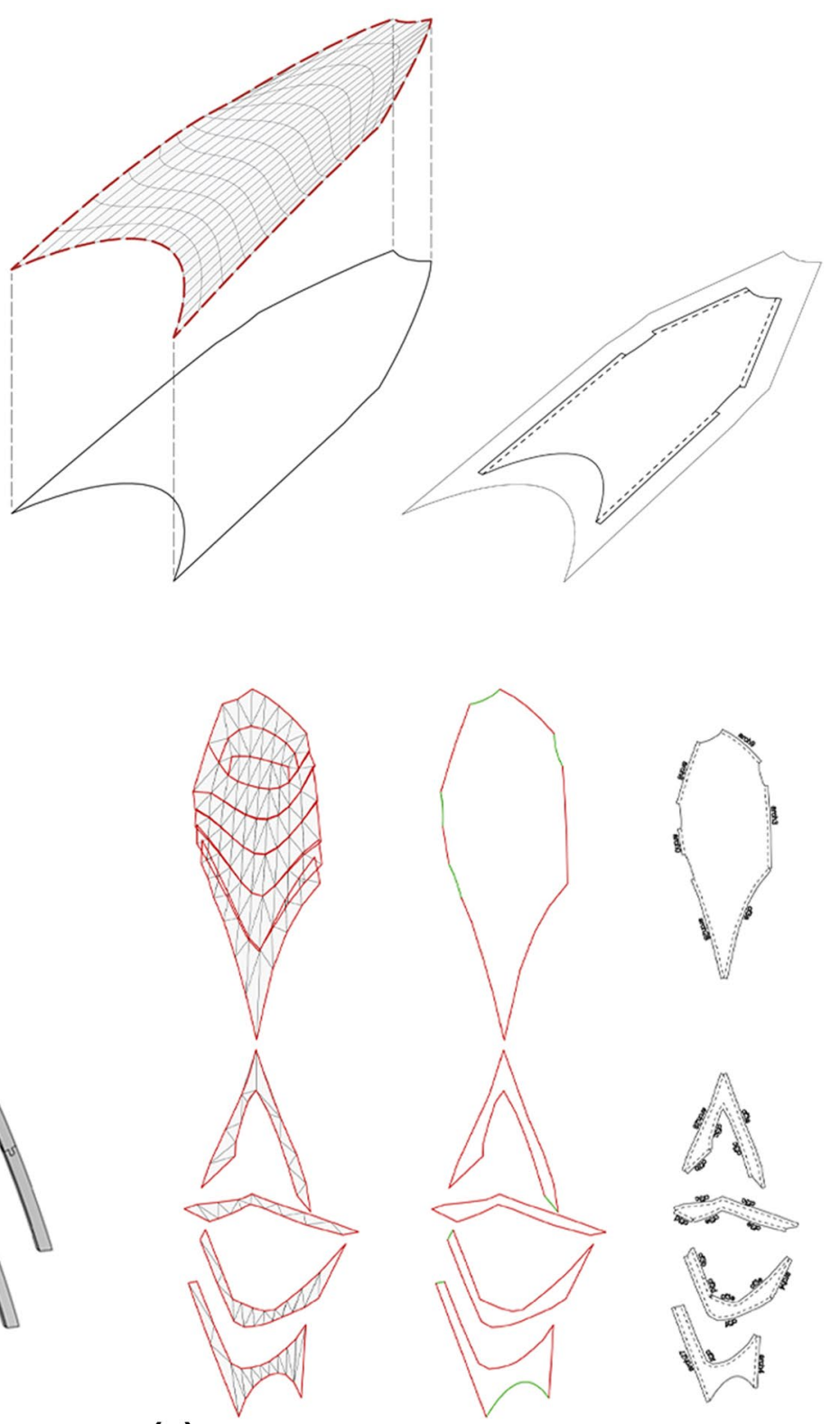

(c)

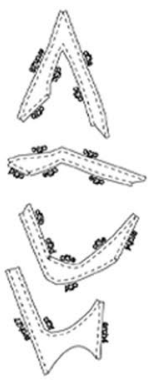

low-resolution mesh then divided into c developable strips with minimised local curvature, subsequently scaled down by $20 \%$ and labelled 
Fig. 16 a Fabric cutting templates labelled and nested on sheets with a grid of $140 \mathrm{~mm}$ spacing $\mathbf{b}$ detail photos of fabric 'funnels'

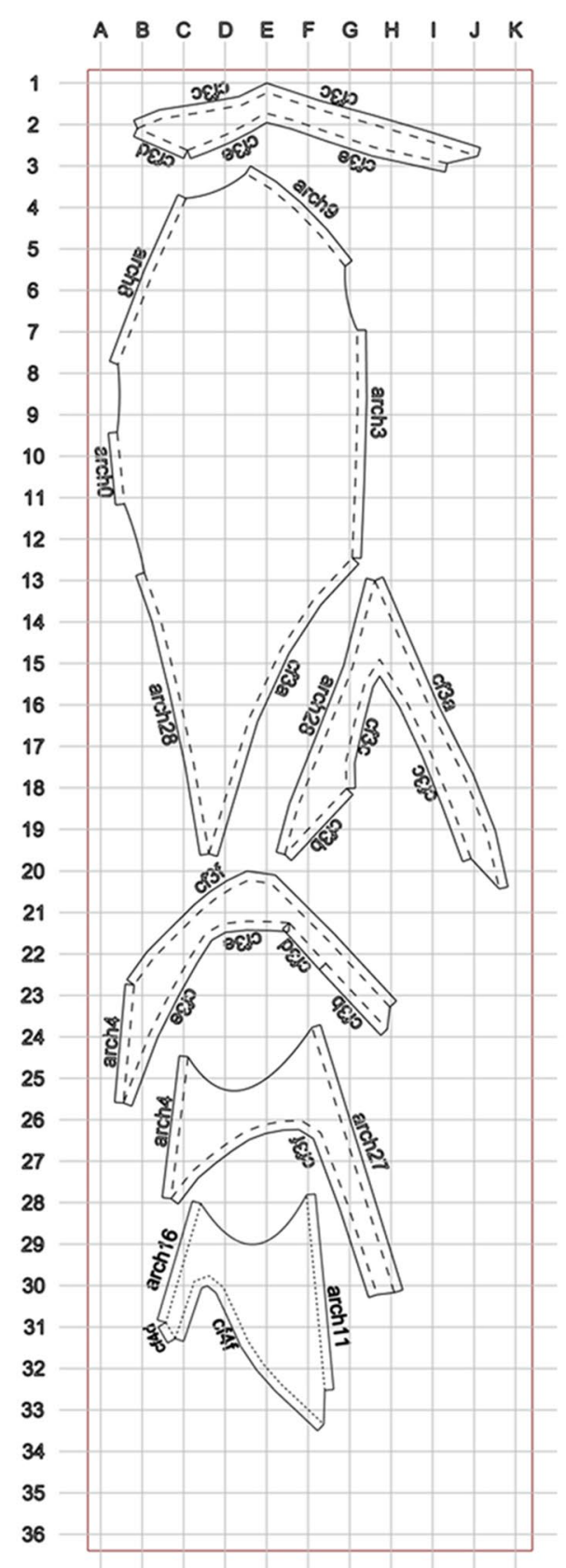

(a)

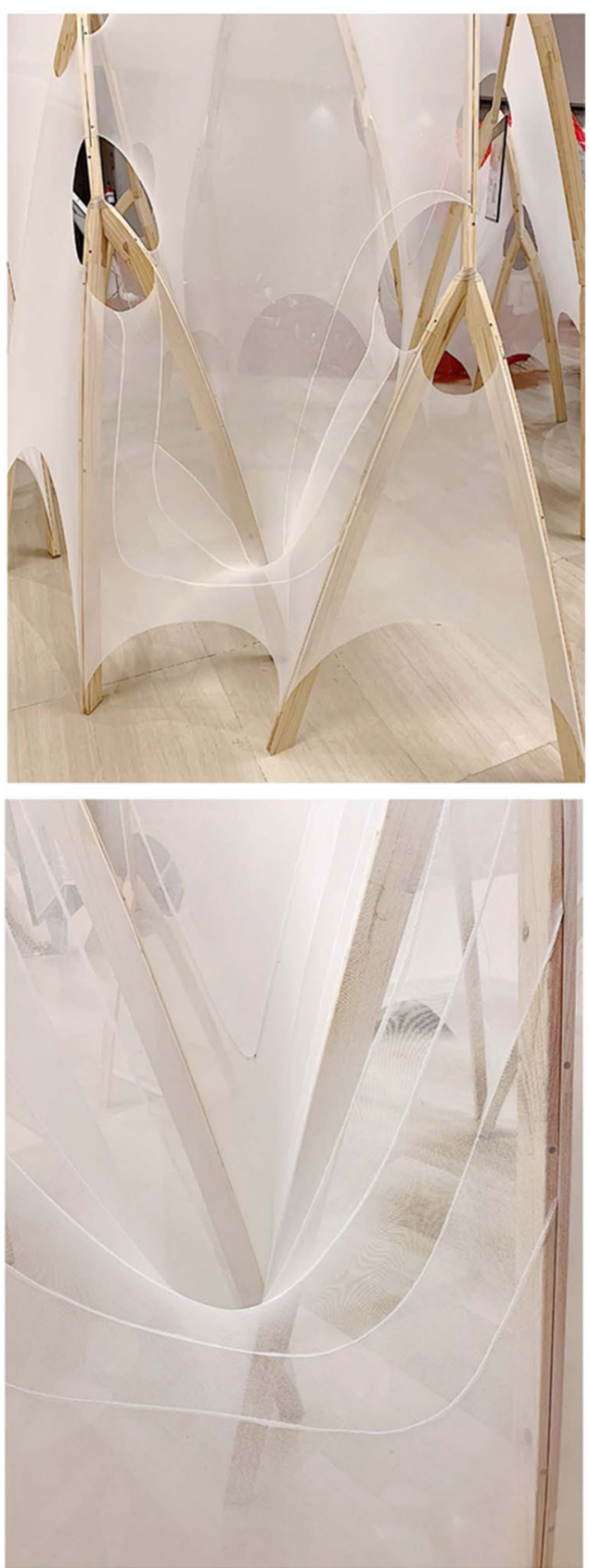

(b) 


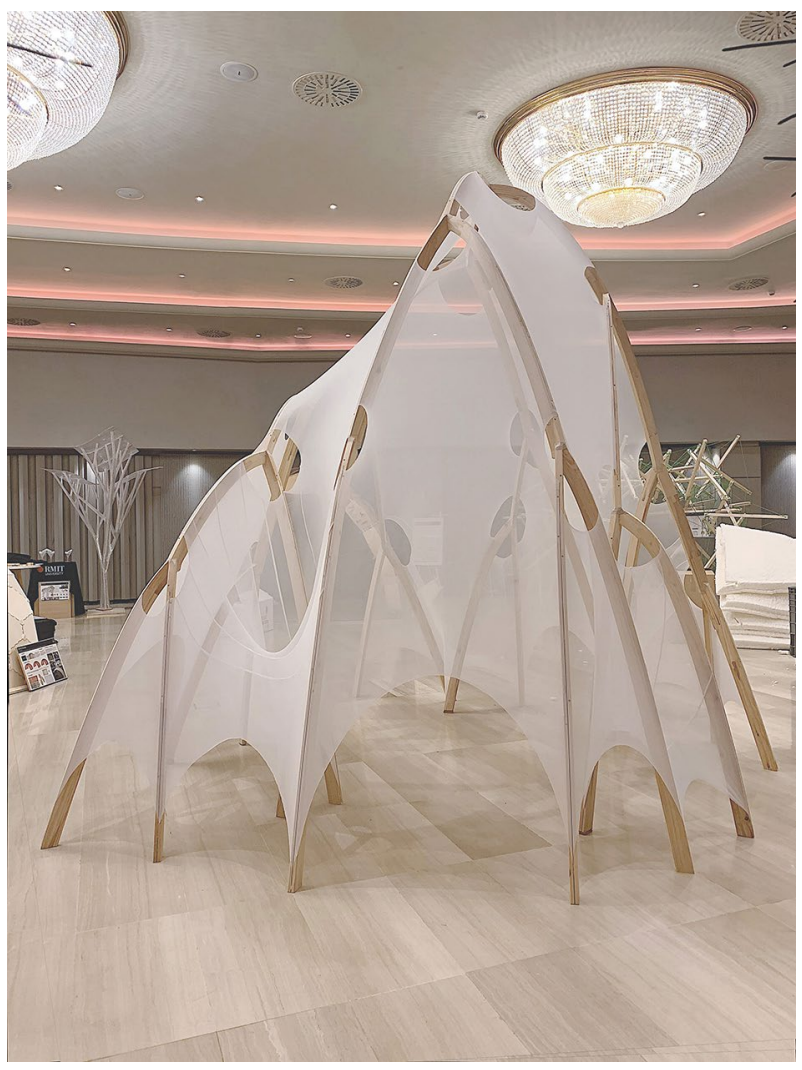

Fig. 17 Completed pavilion installed at the IASS exhibition

\section{Discusssion}

\subsection{Utility-grade timber utilisation}

The realization of the prototype demonstrates a process that can transform utility grade timber into a high-value timber product. From $109 \mathrm{~kg}$ of original utility grade stock, approximately $96.2 \mathrm{~kg}$ was recovered as short length structural grade members, described as $M_{\text {material }}$. Of this, $45.8 \mathrm{~kg}$ was utilized for the final structure member weight, described as $M_{\text {structure. }}$. The final achieved recovery rate, $R R=M_{\text {structure }} / M_{\text {material, }}$ is $47.6 \%$. Of the remaining $M_{\text {material }}$ not used in the final structure, $27.4 \mathrm{~kg}$ (28.5\%) can be accounted for as un-assigned inventory and $23.0 \mathrm{~kg}(23.9 \%)$ as machine waste. The un-assigned inventory included lengths which were too short to be practically deployed in this structure $(<280 \mathrm{~mm} ; 10.7 \mathrm{~kg})$ or available lengths not selected by the part assignment heuristic (17/98 inventory parts; $16.7 \mathrm{~kg})$. The machine waste included sawdust $(12 \mathrm{~mm}$ router bit diameter; $9.4 \mathrm{~kg}$ ) and tolerance allowance $(6-12 \mathrm{~mm}$ internal boundary offset; $13.6 \mathrm{~kg}$ ).

For the scale of the structure the timber funicular gives a very light form, with only $9.2 \mathrm{~kg}$ total node connection weight, $2 \mathrm{~kg}$ of fabric, and $700 \mathrm{~g}$ of metal fixings in addition to the $45.8 \mathrm{~kg}$ of timber member weight. The structure also has inherent value when considering lifecycle costs, due to it being re-deployable and built from renewable material. It can be demonstrated, therefore, that the application of this design process has delivered a spatial structure of very high value, yielded from an under-valued resource.

The amount of material not utilised from the inventory suggest there are opportunities to further increase the resource recovery volume. More specific form controls or optimality conditions could be introduced to ensure all available inventory is assigned; or alternatively parts not used for the present structure could be re-deployed in another project, and therefore not be considered as waste. The latter approach, where a larger pool of available inventory is deliberately set to exceed the aggregate length of required structural elements, has the benefit of enabling a higher fidelity of the part-assigned geometry compared to the target geometry. Improvements to machining tolerance allowance and utilization of very-short lengths (for example as node tail components) would also contribute to an improved recovery rate.

\subsection{Inventory-Constrained Funicular Modelling}

The developed form finding model has been a highly effective interactive design tool. It allowed free exploration of form whilst ensuring a rational configuration of structure and deployment of material. Running the dynamic relaxation simulation, the part assignment algorithm, and the second-round relaxation in rapid succession, enabled the model to provide the user with an immediacy of spatial feedback. A key factor in achieving this rapid processing was the definition of the part assignment as a best-fit heuristic, providing a solution that is achieved very quickly even if potentially sub-optimal. A goal of a perfect fit inventory was 'traded-off' in favor of immediacy in user feedback. In addition, it was found that the funicular geometry provided a latent geometric characteristic that is particularly well suited to a part assignment based on curvature criteria. When starting from its base, a funicular curve will inherently progress from shallow to tighter curvature, correlating to an ever diminishing part length (with a uniform sagitta value), enabling part sorting to have a predictable and efficient sequence.

Finally, it was promising to see the model operate with a high level of spatial responsiveness, with the adjustment of individual chain lengths resulting in near instantaneous global change of form. In this way it can be seen to behave like the physical hanging chain model, providing interaction between spatial, structural and material determinants, but also allowing fast, iterative adjustment of chain length 
based on the specific inventory constraints. The pavilion was awarded a special mention by the judges in the IASS 'Working Group 21' competition awards ceremony.

\section{Conclusion}

The research undertaken in the design and prototyping for this pavilion has provided two key outcomes: a process for increasing the yield of structural grade timber from otherwise under-valued plantation resources; and a design modelling tool that rationalises material usage through a dual process of optimising structural geometry and minimising waste. This is achieved through both structural and material efficiencies, combining the dual constraints of an enforced static equilibrium through funicular geometry, in conjunction with an optimised assignment of an inventory of parts. With the high level of functionality provided in the modelling tool and the process it facilitates, it is anticipated that this research could be applied to the design of larger scale timber structures, and to inventories of other materials.

Acknowledgements This project was funded by the Australian Research Council ARC Future Timber Hub "Industrial Transformation Hub to Transform Tall Timber Buildings" and research industry partners, Hyne Timber are to be thanked for the supply of timber and scan data. The authors would also like to thank those involved in the preparation of the of the "Working Group 21 - Advanced Manufacturing and Materials" exhibition submission, and those who have contributed to preliminary research into the part assignment algorithms, timber fabrication techniques, and presentation material: Daniel Foote, Harry McCullough, Fatemeh Raouf, Nicholas Russel, and Benedikt Blumenröder.

\section{Compliance with ethical standards}

Conflict of interest On behalf of all authors, the corresponding author states that there is no conflict of interest.

\section{References}

1. Ramage $M H$, Burridge $H$, Busse-Wicher $M$, Fereday $G$, Reynolds T, Shah DU, Wu G, Yu L, Fleming P, Densley-Tingley D (2017) The wood from the trees: The use of timber in construction. Renew Sustain Energy Rev 68:333-359

2. Cherry R, Manalo A, Karunasena W, Stringer G (2019) Out-ofgrade sawn pine: $A$ state-of-the-art review on challenges and new opportunities in cross laminated timber (CLT). Constr Build Mater 211:858-868

3. Stotz I, Gouaty G, Weinand Y (2009) Iterative geometric design for architecture. J Int Assoc Shell Spatial Struct 50(1):11-20

4. Apolinarska A, Bärtschi R, Furrer R, Gramazio F, Kohler M (2016) Mastering the sequential roof. Adv Archit Geom 2016:240-258
5. Brütting J, Desruelle J, Senatore G, Fivet C (2019) Design of truss structures through reuse. In: Structures. Elsevier, pp 128-137

6. Bukauskas A, Shepherd P, Walker P, Sharma B, Bregulla J (2017) Form-fitting strategies for diversity-tolerant design. In: Proceedings of IASS annual symposia, vol 17. International Association for Shell and Spatial Structures (IASS), pp 1-10

7. Stanton C (2010) Digitally mediated use of localized material in architecture. In: Paper presented at the Sociedad Iberoamericana de Grafica Digital SIGRADI, Bogota, Colombia

8. Monier V, Bignon JC, Duchanois G (2013) Use of irregular wood components to design non-standard structures. In: Advanced materials research, 2013. Trans Tech Publ, pp 2337-2343

9. Mollica Z, Self M (2016) Tree fork truss. Adv Archit Geom 2016:138-153

10. Bukauskas A, Shepherd P, Walker P, Sharma B, Bregulla J (2018) Inventory-constrained structural design: new objectives and optimization techniques. In: Proceedings of IASS annual symposia, vol 11. International Association for Shell and Spatial Structures (IASS), pp 1-8

11. Tomlow J, Gaudí A (1989) Das modell, vol 34. Institut fur Leichte Flachentragwerke

12. Block PPCV (2009) Thrust network analysis: exploring threedimensional equilibrium. Massachusetts Institute of Technology, Cambridge

13. Kilian A, Ochsendorf J (2005) Particle-spring systems for structural form finding. J Int Assoc Shell Spatial Struct 46(2):77-84

14. Hunt J, Haase W, Sobek W (2009) A design tool for spatial tree structures. J Int Assoc Shell Spatial Struct 50(1):3-10

15. Hensel DS, Bover GB (2015) A developmental route to local specificity. Nested catenaries. Archit Des 85(2):120-127

16. Brütting J, Vandervaeren C, Senatore G, De Temmerman N, Fivet C (2020) Environmental impact minimization of reticular structures made of reused and new elements through life cycle assessment and mixed-integer linear programming. Energy Build 215:109827

17. Bukauskas A (2019) Inventory-constrained structural design (doctoral thesis). University of Bath, Bath, United Kingdom

18. Wang $Y$ (2016) Hooke park biomass boiler house. In: Menges A, Schwinn T, Krieg OD (eds) Advancing wood architecture: a computational approach. Routledge, London

19. Plasencia Alava KB, McCann LK, Hodge G, Baber K, Gattas JM (2019) Computational design and experimental behaviour of deployable mass timber arches. J Int Assoc Shell Spatial Struct 60(1):90-100

20. Gattas J (2018) A generative shape grammar for piecewise cylindrical surfaces and curved-crease origami. In: Lang RJ, Bolitho $M$, You Z (ed) Origami^^7, vol 3. Engineering One. Tarquin Group, pp 921-934

21. Huntington CG (2013) Tensile fabric structures: design, analysis, and construction. American Society of Civil Engineers

22. Fornes $M$ (2014) Double agent white. Paper presented at the ACADIA 14: design agency: the 34th Annual Conference of the Association for Computer Aided Design in Architecture, Los Angeles, 23-25 October, 2014

23. Giannopoulou E, Baquero P, Warang A, Orciuoli A, Estévez AT, Brun-Usan MA (2019) Employing mesh segmentation algorithms as fabrication strategies: Pattern generation based on reaction-diffusion mechanism. FME Trans 47(2):379-386

Publisher's Note Springer Nature remains neutral with regard to jurisdictional claims in published maps and institutional affiliations. 